\title{
TRACE SCALING AUTOMORPHISMS OF THE STABILIZED RAZAK-JACELON ALGEBRA
}

\author{
NORIO NAWATA
}

\begin{abstract}
We classify trace scaling automorphisms of $\mathcal{W} \otimes \mathbb{K}$ up to outer conjugacy, where $\mathcal{W}$ is a certain simple separable nuclear stably projectionless $\mathrm{C}^{*}$-algebra having trivial $K$-groups. Also, we show that all automorphisms of $\mathcal{W}$ with the Rohlin property are outer conjugate to each other. Moreover, we show that the central sequence $\mathrm{C}^{*}$-algebra $F(\mathcal{W})$ of $\mathcal{W}$ is infinite, which answers a question of Kirchberg.
\end{abstract}

\section{INTRODUCTION}

Let $\mathcal{W}$ be the Razak-Jacelon algebra studied in [21, which is a certain simple separable nuclear stably projectionless $\mathrm{C}^{*}$-algebra having trivial $K$-groups and a unique tracial state and no unbounded traces. We may regard $\mathcal{W}$ as a stably finite analogue of the Cuntz algebra $\mathcal{O}_{2}$. Note that a $\mathrm{C}^{*}$-algebra $A$ is said to be stably projectionless if $A \otimes \mathbb{K}$ has no non-zero projections, where $\mathbb{K}$ is the $\mathrm{C}^{*}$-algebra of compact operators on an infinite-dimensional separable Hilbert space. In particular, every stably projectionless $\mathrm{C}^{*}$-algebra is non-unital. We refer the reader to [12, 15 and 16 for remarkable progress in the classification of such $\mathrm{C}^{*}$-algebras.

In this paper, we study trace scaling automorphisms of $\mathcal{W} \otimes \mathbb{K}$ and show that these automorphisms are outer conjugate if and only if the scaling factors coincide. This classification can be regarded as an analogous result of Connes' classification 5 of trace scaling automorphisms of the AFD factor of type $\mathrm{II}_{\infty}$. In the case of $\mathrm{C}^{*}$-algebras, Elliott, Evans and Kishimoto [10] classified trace scaling automorphisms of stable UHF algebras. Moreover, Evans and Kishimoto [13] classified trace scaling automorphisms of stable AF algebras with totally ordered $K_{0}$-groups. (See also 3. 3.) More generally, the study of group actions on operator algebras is one of the most fundamental subjects and has a long history in the theory of operator algebras. We refer the reader to 20 and the references given there for this subject. We recall some other classification results of automorphisms of $\mathrm{C}^{*}$-algebras. Kishimoto 25] showed that if $\alpha$ and $\beta$ are automorphisms of a UHF algebra such that $\alpha^{m}$ and $\beta^{m}$ are strongly outer for any $m \in \mathbb{Z} \backslash\{0\}$, then $\alpha$ and $\beta$ are outer conjugate. Moreover, Kishimoto classified a large class of automorphisms of certain AT algebras in 27] and 28. Matui 35 generalized this result to certain simple AH algebras. Nakamura [4] completely classified aperiodic automorphisms of Kirchberg algebras. Sato 51 showed that if $\alpha$ and $\beta$ are automorphisms of the Jiang-Su algebra $\mathcal{Z}$ such that $\alpha^{m}$ and $\beta^{m}$ are strongly outer for any $m \in \mathbb{Z} \backslash\{0\}$, then $\alpha$ and $\beta$ are outer conjugate. Note that it is important to consider the Rohlin property or variants thereof for classifying automorphisms of operator algebras.

If $A$ is stably projectionless, then the central sequence $\mathrm{C}^{*}$-algebra $A_{\omega}$ of $A$ is also stably projectionless. Hence $A_{\omega}$ is not very useful for our purpose. In this

2010 Mathematics Subject Classification. Primary 46L40, Secondary 46L35; 46L55.

Key words and phrases. Stably projectionless $\mathrm{C}^{*}$-algebra; Trace scaling automorphism; Rohlin property; Kirchberg's central sequence $\mathrm{C}^{*}$-algebra.

This work was supported by JSPS KAKENHI Grant Number 16K17614. 
paper, Kirchberg's central sequence $\mathrm{C}^{*}$-algebra 22 plays a central role. Kirchberg's central sequence $\mathrm{C}^{*}$-algebra $F(A)$ is defined as the quotient $\mathrm{C}^{*}$-algebra of $A_{\omega}$ by the annihilator of $A$.

This paper is organized as follows: In Section 2, we collect notations and some results. In Section 3, we review some results in [12] and reformulate for our purpose. Note that our arguments are essentially based on Elliott and Niu's arguments. In Section 4, we study properties of $F(\mathcal{W})$. We show that $F(\mathcal{W})$ has many projections (Proposition 4.2). This is an easy corollary of Razak's classification theorem [46] and Matui and Sato's result in 39 . But this property enables us to deal with $F(\mathcal{W})$ like a $C^{*}$-algebra of real rank zero. In Section 5. we obtain a homotopy type theorem for unitaries in $F(\mathcal{W})$ by classifying certain unitaries in $F(\mathcal{W})$ up to unitary equivalence (Theorem 5.7, Theorem 5.3). Also, we classify certain projections in $F(\mathcal{W})$ up to unitary equivalence and show that the unit 1 in $F(\mathcal{W})$ is infinite (Theorem 5.8. Corollary 5.9). This is an answer to [22, Question 2.14]. Some arguments in this section are motivated by arguments in [36, Section 4] (see also 32]). In Section 6. we introduce the Rohlin property for automorphisms of separable $\mathrm{C}^{*}$-algebras and show that every trace scaling automorphism of $\mathcal{W} \otimes \mathbb{K}$ has the Rohlin property (Theorem 6.4). Moreover, we show that if $\alpha$ is an automorphism of $\mathcal{W}$ such that $\alpha^{m}$ is strongly outer for any $m \in \mathbb{Z} \backslash\{0\}$, then $\alpha$ has the Rohlin property (Theorem 6.7). In Section 7, we obtain a classification theorem (Theorem 7.3) of trace scaling automorphisms of $\mathcal{W} \otimes \mathbb{K}$ by using the Bratteli-Elliott-Evans-Kishimoto intertwining argument. By the uniqueness of traces on $\mathcal{W} \otimes \mathbb{K}$, for any automorphism $\alpha$ of $\mathcal{W} \otimes \mathbb{K}$, there exists a positive real number $\lambda(\alpha)$ such that $\tau \otimes \operatorname{Tr} \circ \alpha=\lambda(\alpha) \tau \otimes \operatorname{Tr}$. We say that $\alpha$ is a trace scaling automorphism if $\lambda(\alpha) \neq 1$. The following theorem is the main result in this paper.

\section{Theorem.}

Let $\alpha$ and $\beta$ be trace scaling automorphisms of $\mathcal{W} \otimes \mathbb{K}$. Then $\alpha$ and $\beta$ are outer conjugate if and only if $\lambda(\alpha)=\lambda(\beta)$.

The range of the invariant $\left\{\lambda(\alpha) \in \mathbb{R}_{+}^{\times} \mid \alpha \in \operatorname{Aut}(\mathcal{W} \otimes \mathbb{K})\right\}$ is equal to the fundamental group $\mathcal{F}(\mathcal{W})$ of $\mathcal{W}$, which is introduced in 42 , (see also [43. Proposition 2.8]). By Razak's classification theorem 46 and Robert's classification theorem [47, $\mathcal{F}(\mathcal{W})$ is equal to $\mathbb{R}_{+}^{\times}$. Moreover, combining the results of Kishimoto-Kumjian 30, 31, Dean 9] and Robert [47, we see that there exists a trace scaling flow on $\mathcal{W} \otimes \mathbb{K}$. Note that a separable $\mathrm{C}^{*}$-algebra with the uncountable fundamental group must be stably projectionless (see [42, Corollary 4.10]). Hence stably projectionless $\mathrm{C}^{*}$-algebras seem to be more analogous to the AFD factors of type II than (finite) stably unital $\mathrm{C}^{*}$-algebras. We also show that if $\alpha$ and $\beta$ are automorphisms of $\mathcal{W}$ such that $\alpha^{m}$ and $\beta^{m}$ are strongly outer for any $m \in \mathbb{Z} \backslash\{0\}$, then $\alpha$ and $\beta$ are outer conjugate (Theorem 7.4 ).

After the first version of this paper was on the arxiv, Gábor Szabó generalized some results in this paper to all classifiable $K K$-contractible $\mathrm{C}^{*}$-algebras (see 53 , Theorem 5.11 and Theorem 5.12]) by using Gong and Lin's basic homotopy lemma 15.

\section{Preliminaries}

In this section we shall collect notations and some results. We refer the reader to [1] and 45] for basic facts of operator algebras.

2.1. Notation. We say that a $\mathrm{C}^{*}$-algebra $A$ is $\sigma$-unital if $A$ has a countable approximate unit. Note that if $A$ is separable, then $A$ is $\sigma$-unital. If $A$ is $\sigma$-unital, then there exists a positive element $s \in A$ such that $\left\{s^{\frac{1}{n}}\right\}_{n \in \mathbb{N}}$ is an approximate unit. Such a positive element $s$ is called strictly positive in $A$. We denote by $A^{\sim}$ 
the unitization algebra of $A$. The multiplier algebra, denoted by $M(A)$, of $A$ is the largest unital $\mathrm{C}^{*}$-algebra that contains $A$ as an essential ideal. If $\alpha$ is an automorphism of $A$, then $\alpha$ extends uniquely to an automorphism of $M(A)$. We denote it by the same symbol $\alpha$ for simplicity.

For a unitary element $u$ in $M(A)$, define an automorphism $\operatorname{Ad}(u)$ of $A$ by $\operatorname{Ad}(u)(x)=u x u^{*}$ for $x \in A$. Such an automorphism is called an inner automorphism. Let $\operatorname{Aut}(A)$ denote the automorphism group of $A$, which is equipped with the topology of pointwise norm convergence. An automorphism $\alpha$ is said to be approximately inner if $\alpha$ is in the closure of the inner automorphism group. We say that two automorphisms $\alpha$ and $\beta$ are approximately unitarily equivalent if $\alpha \circ \beta^{-1}$ is approximately inner, and are outer conjugate if there exist an automorphism $\gamma$ of $A$ and a unitary element $u$ in $M(A)$ such that

$$
\alpha=\operatorname{Ad}(u) \circ \gamma \circ \beta \circ \gamma^{-1} \text {. }
$$

Let $F$ be a subset of $A$ and $\varepsilon>0$. A completely positive (c.p.) map $\varphi: A \rightarrow B$ is said to be $(F, \varepsilon)$-multiplicative if

$$
\|\varphi(x y)-\varphi(x) \varphi(y)\|<\varepsilon
$$

for any $x, y \in F$. For c.p. maps $\varphi, \psi: A \rightarrow B$, we write $\varphi \sim_{F, \varepsilon} \psi$ if there exists a unitary element $u \in B^{\sim}$ such that

$$
\left\|\varphi(x)-u \psi(x) u^{*}\right\|<\varepsilon
$$

for any $x \in F$.

We denote by $A_{+}$the set of positive elements in $A$ and by $A_{+, 1}$ the set of positive contractions in $A$. A trace on $A$ is a map $\tau$ of $A_{+}$to $[0, \infty]$ such that $\tau(\lambda a)=\lambda \tau(a)$, $\tau(a+b)=\tau(a)+\tau(b)$ and $\tau\left(x^{*} x\right)=\tau\left(x x^{*}\right)$ for any $a, b \in A_{+}, \lambda \geq 0$ and $x \in A$. For a trace $\tau$ on $A$, let $\mathfrak{M}_{\tau}$ be a linear span of $\left\{a \in A_{+} \mid \tau(a)<\infty\right\}$ and $\mathfrak{N}_{\tau}:=\{x \in A$ : $\left.\tau\left(x^{*} x\right)<\infty\right\}$. Then $\mathfrak{M}_{\tau}$ and $\mathfrak{N}_{\tau}$ are ideals of $A$ and $\tau$ can be uniquely extended to a positive linear functional on $\mathfrak{M}_{\tau}$. A tracial state is a trace which is a state. Every tracial state on $A$ extends uniquely to a tracial state on $M(A)$. We denote it by the same symbol for simplicity. We say that $\tau$ is densely defined if $\mathfrak{M}_{\tau}$ is dense in $A$, and is lower semicontinuous if $\left\{a \in A_{+} \mid \tau(a) \leq r\right\}$ is closed for any $r \in \mathbb{R}_{+}$. Let $T(A)$ denote the set of densely defined lower semicontinuous traces on $A$ and $T_{1}(A)$ the set of tracial states on $A$. For $\tau \in T(A)$, put $d_{\tau}(a)=\lim _{n \rightarrow \infty} \tau\left(a^{\frac{1}{n}}\right)$ for $a \in A_{+}$. Then $d_{\tau}$ is a dimension function. We denote by $\left(\pi_{\tau}, H_{\tau}\right)$ the GelfandNaimark-Segal (GNS) representation of $\tau \in T(A)$. Note that $H_{\tau}$ is the completion of the pre-Hilbert space $\mathfrak{N}_{\tau}$ with a pre-inner product $\langle\widehat{x}, \widehat{y}\rangle=\tau\left(y^{*} x\right)$ for $x, y \in \mathfrak{N}_{\tau}$. The norm on $H_{\tau}$ is denoted by $\|\cdot\|_{2}$. Let $\tau$ be a lower semicontinuous densely defined trace on a $\sigma$-unital $\mathrm{C}^{*}$-algebra $A$. We denote by $\operatorname{Ped}(A)$ the Pedersen ideal of $A$, which is a minimal dense ideal of $A$. Note that $\operatorname{Ped}(A)$ is contained in $\mathfrak{N}_{\tau}$ because $\mathfrak{N}_{\tau}$ is a dense ideal in $A$. There exists an approximate unit $\left\{h_{n}\right\}_{n \in \mathbb{N}}$ for $A$ contained in $\operatorname{Ped}(A)$. It easy to see that $\left\{\widehat{h_{n} x} \mid n \in \mathbb{N}, x \in \mathfrak{N}_{\tau}\right\}$ is dense in $H_{\tau}$. Indeed, the lower semicontinuity of $\tau$ implies that for any $x \in \mathfrak{N}_{\tau}$, we have

$$
\left\|\widehat{x}-\widehat{h_{n} x}\right\|_{2}=\tau\left(x^{*}\left(1-h_{n}\right)^{2} x\right)^{\frac{1}{2}} \leq \tau\left(x^{*}\left(1-h_{n}\right) x\right)^{\frac{1}{2}} \rightarrow 0
$$

as $n \rightarrow \infty$. If $\alpha$ is an automorphism of $A$ such that $\tau \circ \alpha=\lambda \tau$ for some $\lambda \in \mathbb{R}_{+}^{\times}$, then $\alpha$ can be uniquely extended to an automorphism $\tilde{\alpha}$ of $\pi_{\tau}(A)^{\prime \prime}$.

For $x, y \in A$, we write $[x, y]$ to mean the commutator $x y-y x$. We denote by $\mathbb{K}$ and $M_{n \infty}$ for $n \in \mathbb{N}$ the $\mathrm{C}^{*}$-algebra of compact operators on an infinite-dimensional separable Hilbert space and the uniformly hyperfinite (UHF) algebra of type $n^{\infty}$, respectively. Let $\operatorname{Tr}_{n}$ for $n \in \mathbb{N}$ denote the (unnormalized) usual trace on $M_{n}(\mathbb{C})$ and $\operatorname{Tr}$ denote the usual trace on $\mathbb{K}$. 
2.2. Kirchberg's central sequence $\mathbf{C}^{*}$-algebras. We shall recall some properties of Kirchberg's central sequence $\mathrm{C}^{*}$-algebras in [22] (see also [43, Section 5]). Let $\omega$ be a free ultrafilter on $\mathbb{N}$. For a $\sigma$-unital $\mathrm{C}^{*}$-algebra $A$, set

$$
c_{\omega}(A):=\left\{\left(x_{n}\right)_{n \in \mathbb{N}} \in \ell^{\infty}(\mathbb{N}, A) \mid \lim _{n \rightarrow \omega}\left\|x_{n}\right\|=0\right\}, A^{\omega}:=\ell^{\infty}(\mathbb{N}, A) / c_{\omega}(A) .
$$

Let $B$ be a $\mathrm{C}^{*}$-subalgebra of $A$. We identify $A$ and $B$ with the $\mathrm{C}^{*}$-subalgebras of $A^{\omega}$ consisting of equivalence classes of constant sequences. Put

$$
A_{\omega}:=A^{\omega} \cap A^{\prime}, \operatorname{Ann}\left(B, A^{\omega}\right):=\left\{\left(x_{n}\right)_{n} \in A^{\omega} \cap B^{\prime} \mid\left(x_{n}\right)_{n} b=0 \text { for any } b \in B\right\} .
$$

Then $\operatorname{Ann}\left(B, A^{\omega}\right)$ is a closed ideal of $A^{\omega} \cap B^{\prime}$, and define

$$
F(A):=A_{\omega} / \operatorname{Ann}\left(A, A^{\omega}\right) .
$$

We call $F(A)$ the central sequence $C^{*}$-algebra of $A$. A sequence $\left(x_{n}\right)_{n}$ is said to be central if $\lim _{n \rightarrow \omega}\left\|\left[x_{n}, x\right]\right\|=0$ for all $x \in A$. A central sequence is a representative of an element in $A_{\omega}$. Since $A$ is $\sigma$-unital, $A$ has a countable approximate unit $\left\{h_{n}\right\}_{n \in \mathbb{N}}$. It is easy to see that $\left[\left(h_{n}\right)_{n}\right]$ is a unit in $F(A)$. If $A$ is unital, then $F(A)=A_{\omega}$. Note that $F(A)$ is isomorphic to $M(A)^{\omega} \cap A^{\prime} / \operatorname{Ann}\left(A, M(A)^{\omega}\right)$ and $A^{\sim}{ }_{\omega} / \operatorname{Ann}\left(A,\left(A^{\sim}\right)^{\omega}\right)$. Indeed, for any $\left(y_{n}\right)_{n} \in M(A)^{\omega} \cap A^{\prime}$ (respectively, $\left.\left(y_{n}\right)_{n} \in A^{\sim} \omega\right),\left(y_{n} h_{n}\right)_{n}$ is a central sequence in $A$ and $\left[\left(y_{n}\right)_{n}\right]=\left[\left(y_{n} h_{n}\right)_{n}\right]$ in $M(A)^{\omega} \cap A^{\prime} / \operatorname{Ann}\left(A, M(A)^{\omega}\right)$ (respectively, in $A^{\sim}{ }_{\omega} / \operatorname{Ann}\left(A,\left(A^{\sim}\right)^{\omega}\right)$ ). Let $h$ be a full positive element in $A$, and define a map $\eta$ from $F(A)$ to $F(\overline{h A h})$ by $\eta\left(\left[\left(x_{n}\right)_{n}\right]\right)=$ $\left[\left(h^{\frac{1}{n}} x_{n} h^{\frac{1}{n}}\right)_{n}\right]$. Then $\eta$ is an isomorphism from $F(A)$ onto $F(\overline{h A h})$. In particular, $F(A \otimes \mathbb{K})$ is isomorphic to $F(A)$. If $\alpha$ is an automorphism of $A, \alpha$ induces natural automorphisms of $A^{\omega}, A_{\omega}$ and $F(A)$. We denote them by the same symbol $\alpha$ for simplicity.

There exists a natural homomorphism $\rho$ from $F(A) \otimes_{\max } A$ to $A^{\omega}$ such that

$$
\rho\left(\left[\left(x_{n}\right)_{n}\right] \otimes x\right)=\left(x_{n} x\right)_{n}
$$

for any $\left[\left(x_{n}\right)_{n}\right] \in F(A)$ and $x \in A$. For a projection $p$ in $F(A)$, let $A_{p}^{\omega}$ be a hereditary subalgebra of $A^{\omega}$ generated by $\rho\left(p F(A) p \otimes_{\max } A\right)$. It can be easily checked that

$$
A_{p}^{\omega}=\overline{\rho(p \otimes s) A^{\omega} \rho(p \otimes s)}
$$

where $s$ is a strictly positive element in $A$.

Since $\omega$ is a (free) ultrafilter, for any $\tau \in T_{1}(A)$, we can define a tracial state $\tau_{\omega}$ on $A^{\omega}$ by $\tau_{\omega}\left(\left(a_{n}\right)_{n}\right)=\lim _{n \rightarrow \omega} \tau\left(a_{n}\right)$ for any $\left(a_{n}\right)_{n} \in A^{\omega}$. We shall show that $\tau_{\omega}$ is well-defined on $F(A)$.

Proposition 2.1. Let $A$ be a $\sigma$-unital $\mathrm{C}^{*}$-algebra, and let $\tau$ be a tracial state on $A$. Define $\tau_{\omega}: F(A) \rightarrow \mathbb{C}$ by

$$
\tau_{\omega}\left(\left[\left(x_{n}\right)_{n}\right]\right)=\lim _{n \rightarrow \omega} \tau\left(x_{n}\right)
$$

for any $\left[\left(x_{n}\right)_{n}\right] \in F(A)$. Then $\tau_{\omega}$ is well-defined. In particular, $\tau_{\omega}$ is a tracial state on $F(A)$.

Proof. It suffices to show that if $\left(x_{n}\right)_{n} \in \operatorname{Ann}\left(A, A^{\omega}\right)$, then $\lim _{n \rightarrow \omega} \tau\left(x_{n}\right)=0$. We may assume that $\left\|x_{n}\right\| \leq 1$ for any $n \in \mathbb{N}$. Let $\left\{h_{n}\right\}_{n \in \mathbb{N}}$ be an approximate unit for $A$ and $\varepsilon>0$. There exists a natural number $N$ such that

$$
\left|1-\tau\left(h_{N}\right)\right|<\frac{\varepsilon}{2}
$$

because $\lim _{n \rightarrow \infty} \tau\left(h_{n}\right)=1$. Since $\lim _{n \rightarrow \omega}\left\|x_{n} h_{N}\right\|=0$, there exists $X \in \omega$ such that

$$
\left|\tau\left(x_{n} h_{N}\right)\right|<\frac{\varepsilon}{2}
$$


for any $n \in X$. Hence we have

$$
\begin{aligned}
\left|\tau\left(x_{n}\right)\right| & \leq\left|\tau\left(x_{n}\right)-\tau\left(x_{n} h_{N}\right)\right|+\left|\tau\left(x_{n} h_{N}\right)\right|<\left|\tau\left(\left(1-h_{N}\right)^{\frac{1}{2}} x_{n}\left(1-h_{N}\right)^{\frac{1}{2}}\right)\right|+\frac{\varepsilon}{2} \\
& \leq\left|1-\tau\left(h_{N}\right)\right|+\frac{\varepsilon}{2}<\varepsilon
\end{aligned}
$$

for any $n \in X$. Therefore $\lim _{n \rightarrow \omega} \tau\left(x_{n}\right)=0$.

For a semifinite von Neumann algebra $M$ with separable predual, set $\mathscr{C}_{\omega}(M):=\left\{\left(x_{n}\right)_{n \in \mathbb{N}} \in \ell^{\infty}(\mathbb{N}, M) \mid\left[x_{n}, y\right] \rightarrow 0 *\right.$-strongly as $n \rightarrow \omega$ for any $\left.y \in M\right\}$ and

$$
\mathscr{T}_{\omega}(M):=\left\{\left(x_{n}\right)_{n \in \mathbb{N}} \in \ell^{\infty}(\mathbb{N}, M) \mid x_{n} \rightarrow 0 * \text {-strongly as } n \rightarrow \omega\right\} .
$$

Then $\mathscr{C}_{\omega}(M)$ is a $\mathrm{C}^{*}$-subalgebra of $\ell^{\infty}(\mathbb{N}, M)$ and $\mathscr{T}_{\omega}(M)$ is a closed ideal of $\mathscr{C}_{\omega}(M)$. Define

$$
M_{\omega}:=\mathscr{C}_{\omega}(M) / \mathscr{T}_{\omega}(M) .
$$

Note that $M_{\omega}$ coincides with the asymptotic centralizer of $M$ in [4, and hence $M_{\omega}$ is a finite von Neumann algebra. (Indeed, [54, Lemma XIV.3.4] and some arguments on semifinite von Neumann algebras show this fact.) Let $p$ be a projection in $M$ with central support 1 , and define a map $\tilde{\eta}$ from $M_{\omega}$ to $(p M p)_{\omega}$ by $\tilde{\eta}\left(\left(x_{n}\right)_{n}\right)=\left(p x_{n} p\right)_{n}$. Then $\tilde{\eta}$ is an isomorphism from $M_{\omega}$ onto $(p M p)_{\omega}$ by [4, Lemma 2.11] (see also [33. Lemma 2.8]) and [33, Proposition 2.10]. If $\alpha$ is an automorphism of a semifinite von Neumann algebra $M, \alpha$ induces a natural automorphism of $M_{\omega}$. We denote it by the same symbol $\alpha$ for simplicity. Note that if $\tau$ is a bounded trace, then the following proposition is clear.

Proposition 2.2. Let $A$ be a $\sigma$-unital $\mathrm{C}^{*}$-algebra, and let $\tau$ be a lower semicontinuous densely defined trace on $A$. Then the inclusion map from $A$ to $\pi_{\tau}(A)^{\prime \prime}$ induces a homomorphism $\varrho_{A}$ from $F(A)$ to $\pi_{\tau}(A)_{\omega}^{\prime \prime}$.

Proof. Let $\left\{h_{m}\right\}_{m \in \mathbb{N}}$ be an approximate unit for $A$ contained in $\operatorname{Ped}(A)$. First, we shall show that if $\left(x_{n}\right)_{n} \in \operatorname{Ann}\left(A, A^{\omega}\right)$, then $\left(\pi_{\tau}\left(x_{n}\right)\right)_{n} \in \mathscr{T}_{\omega}\left(\pi_{\tau}(A)^{\prime \prime}\right)$. For any $m \in \mathbb{N}$ and $x \in \mathfrak{N}_{\tau}$, we have

$$
\left\|\pi_{\tau}\left(x_{n}\right) \widehat{h_{m} x}\right\|_{2}+\left\|\pi_{\tau}\left(x_{n}^{*}\right) \widehat{h_{m} x}\right\|_{2} \leq\left\|x_{n} h_{m}\right\| \cdot\|\widehat{x}\|_{2}+\left\|x_{n}^{*} h_{m}\right\| \cdot\|\widehat{x}\|_{2} \rightarrow 0
$$

as $n \rightarrow \omega$. Hence we see that $\left(\pi_{\tau}\left(x_{n}\right)\right)_{n} \in \mathscr{T}_{\omega}\left(\pi_{\tau}(A)^{\prime \prime}\right)$.

Let $\left(x_{n}\right)_{n}$ be a central sequence of contractions in $A$. If we prove $\left(\pi_{\tau}\left(x_{n}\right)\right)_{n} \in$ $\mathscr{C}_{\omega}\left(\pi_{\tau}(A)^{\prime \prime}\right)$, then we obtain the conclusion. Let $\varepsilon>0$ and $y \in \pi_{\tau}(M)^{\prime \prime}$. It suffices to show that for any $m \in \mathbb{N}$ and $x \in \mathfrak{N}_{\tau}$, there exists $X \in \omega$ such that

$$
\left\|\left[\pi_{\tau}\left(x_{n}\right), y\right] \widehat{h_{m} x}\right\|_{2}+\left\|\left[\pi_{\tau}\left(x_{n}\right), y\right]^{*} \widehat{h_{m} x}\right\|_{2}<\varepsilon
$$

for any $n \in X$. We may assume that $y$ is a contraction and $\widehat{x} \neq 0$. (Note that we also have $x \neq 0$.) By the Kaplansky density theorem, there exists a contraction $z \in A$ such that

$$
\left\|\left(y-\pi_{\tau}(z)\right) \widehat{h_{m}}\right\|_{2}+\left\|\left(y^{*}-\pi_{\tau}\left(z^{*}\right)\right) \widehat{h_{m}}\right\|_{2}<\frac{\varepsilon}{12\|x\|} .
$$

Since $\left(x_{n}\right)_{n}$ is a central sequence in $A$, there exists $X \in \omega$ such that

$$
\left\|\left[x_{n}, z\right]\right\|<\frac{\varepsilon}{6\|\widehat{x}\|_{2}}, \quad\left\|\left[x_{n}, h_{m}\right]\right\|<\frac{\varepsilon}{12\|\widehat{x}\|_{2}}
$$


for any $n \in X$. Then we have

$$
\begin{aligned}
\left\|\left[\pi_{\tau}\left(x_{n}\right), y\right] \widehat{h_{m} x}\right\|_{2} & \leq\left\|\left(\pi_{\tau}\left(x_{n}\right) y-\pi_{\tau}\left(x_{n} z\right)\right) \widehat{h_{m} x}\right\|_{2}+\left\|\left(\pi_{\tau}\left(x_{n} z\right)-y \pi_{\tau}\left(x_{n}\right)\right) \widehat{h_{m} x}\right\|_{2} \\
& \leq\left\|x_{n}\right\| \cdot\left\|\left(y-\pi_{\tau}(z)\right) \widehat{h_{m}}\right\|_{2} \cdot\|x\|+\left\|\left(\pi_{\tau}\left(x_{n} z\right)-y \pi_{\tau}\left(x_{n}\right)\right) \widehat{h_{m} x}\right\|_{2} \\
& <\frac{\varepsilon}{12}+\left\|\pi_{\tau}\left(\left[x_{n}, z\right]\right) \widehat{h_{m} x}\right\|_{2}+\left\|\left(\pi_{\tau}\left(z x_{n}\right)-y \pi_{\tau}\left(x_{n}\right)\right) \widehat{h_{m} x}\right\|_{2} \\
& \leq \frac{\varepsilon}{12}+\left\|\left[x_{n}, z\right]\right\| \cdot\left\|h_{m}\right\| \cdot\|\widehat{x}\|_{2}+\left\|\left(\pi_{\tau}\left(z x_{n}\right)-y \pi_{\tau}\left(x_{n}\right)\right) \widehat{h_{m} x}\right\|_{2} \\
& <\frac{\varepsilon}{4}+\left\|\pi_{\tau}\left(z\left[x_{n}, h_{m}\right]\right) \widehat{x}\right\|_{2}+\left\|\pi_{\tau}\left(z h_{m} x_{n}\right) \widehat{x}-y \pi_{\tau}\left(x_{n}\right) \widehat{h_{m} x}\right\|_{2} \\
& \leq \frac{\varepsilon}{4}+\|z\| \cdot\left\|\left[x_{n}, h_{m}\right]\right\| \cdot\|\widehat{x}\|_{2}+\left\|\left(\pi_{\tau}\left(z h_{m} x_{n}\right)-y \pi_{\tau}\left(x_{n} h_{m}\right)\right) \widehat{x}\right\|_{2} \\
& <\frac{\varepsilon}{3}+\left\|\pi_{\tau}\left(z h_{m} x_{n}\right) \widehat{x}-y \pi_{\tau}\left(h_{m} x_{n}\right) \widehat{x}\right\|_{2}+\left\|y \pi_{\tau}\left(\left[h_{m}, x_{n}\right]\right) \widehat{x}\right\|_{2} \\
& \leq \frac{\varepsilon}{3}+\left\|\left(\pi_{\tau}(z)-y\right) \widehat{h_{m}}\right\|_{2} \cdot\left\|x_{n}\right\| \cdot\|x\|+\|y\| \cdot\left\|\left[h_{m}, x_{n}\right]\right\| \cdot\|\widehat{x}\|_{2} \\
& <\frac{\varepsilon}{2}
\end{aligned}
$$

for any $n \in X$. Similar arguments show

$$
\left\|\left[\pi_{\tau}\left(x_{n}\right), y\right]^{*} \widehat{h_{m} x}\right\|_{2}<\frac{\varepsilon}{2}
$$

for any $n \in X$. Therefore the proof is complete.

It is easy to see that if $\tau$ is a bounded faithful trace on $A$, then $\operatorname{ker}\left(\varrho_{A}\right)=$ $\left\{\left[\left(x_{n}\right)_{n}\right] \in F(A) \mid \lim _{n \rightarrow \omega} \tau\left(x_{n}^{*} x_{n}\right)=0\right\}$. Moreover, the same proof as [24, Theorem 3.3] shows that $\varrho_{A}$ is surjective. (See also [52, Lemma 2.1].) Using this fact, we show the following proposition.

Proposition 2.3. Let $A$ be a $\sigma$-unital $\mathrm{C}^{*}$-algebra, and let $\tau$ be a faithful lower semicontinuous densely defined trace on $A$. Assume that $\operatorname{Ped}(A)$ contains a full positive element. Then $\varrho_{A}$ is surjective.

Proof. Let $h$ be a full positive element in $\operatorname{Ped}(A)$. Then $\overline{h A h}$ is a $\mathrm{C}^{*}$-algebra with no unbounded traces by the same argument as in the proof of 43 , Proposition 5.2]. In particular, $\left.\tau\right|_{\overline{h A h}}$ is a bounded faithful trace on $\overline{h A h}$. Let $p$ be a support projection of $\pi_{\tau}(h)$ in $\pi_{\tau}(A)^{\prime \prime}$. Note that $\pi_{\tau}\left(h^{\frac{1}{n}}\right)$ converges to $p$ in the strong* topology as $n \rightarrow \infty$. Since $h$ is full in $A, p$ has central support 1 . Hence we have the following commutative diagram:

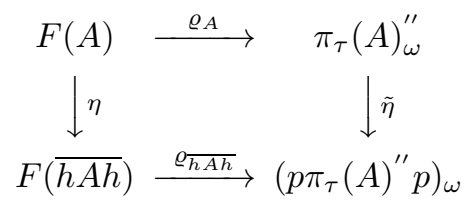

where $\eta$ and $\tilde{\eta}$ are standard isomorphisms from $F(A)$ onto $F(\overline{h A h})$ and from $\pi_{\tau}(A)_{\omega}^{\prime \prime}$ onto $\left(p \pi_{\tau}(A)^{\prime \prime} p\right)_{\omega}$, respectively. Since $\varrho_{\overline{h A h}}$ is surjective, we see that $\varrho_{A}$ is surjective.

Remark 2.4. Let $A$ be a $\sigma$-unital simple $\mathrm{C}^{*}$-algebra, and let $\tau$ be a lower semicontinuous densely defined trace on $A$. Then $\operatorname{Ped}(A)$ contains a full positive element since every non-zero positive element is full. Moreover, it can be easily checked that $\operatorname{ker}\left(\varrho_{A}\right)=\left\{\left[\left(x_{n}\right)_{n}\right] \in F(A) \mid \lim _{n \rightarrow \omega}\left\|\pi_{\tau}\left(x_{n}\right) \hat{h}\right\|_{2}=0\right\}$ for some $h \in \operatorname{Ped}(A)_{+} \backslash\{0\}$. Assume that $\tau$ is a unique (up to scalar multiple) trace. Then we can define a tracial state $\tau_{\omega}$ on $F(A)$ by $\tau_{\omega}\left(\left[\left(x_{n}\right)_{n}\right]\right):=\lim _{n \rightarrow \omega} \tau\left(x_{n} h\right) / \tau(h)$. Note that if $\left[\left(x_{n}\right)_{n}\right] \in \operatorname{ker}\left(\varrho_{A}\right)$, then $\tau_{\omega}\left(\left[\left(x_{n}\right)_{n}\right]\right)=0$. 
2.3. Matui and Sato's result. We shall remark that some arguments in 39 work for non-unital $\mathrm{C}^{*}$-algebras. It is important to consider property (SI). For $a, b \in A_{+}$, we say that $a$ is Cuntz smaller than $b$, written $a \precsim b$, if there exists a sequence $\left\{x_{n}\right\}_{n \in \mathbb{N}}$ of $A$ such that $\left\|x_{n}^{*} b x_{n}-a\right\| \rightarrow 0$.

Definition 2.5. Let $A$ be a $\mathrm{C}^{*}$-algebra.

(1) Assume that $T_{1}(A)$ is a non-empty compact set. We say that $A$ has property (SI) if for any central sequences $\left(a_{n}\right)_{n}$ and $\left(b_{n}\right)_{n}$ of positive contractions in $A$ satisfying

$$
\lim _{n \rightarrow \omega} \max _{\tau \in T_{1}(A)} \tau\left(a_{n}\right)=0, \inf _{m \in \mathbb{N}} \lim _{n \rightarrow \omega} \min _{\tau \in T_{1}(A)} \tau\left(b_{n}^{m}\right)>0,
$$

there exists a central sequence $\left(s_{n}\right)_{n}$ in $A$ such that

$$
\lim _{n \rightarrow \omega}\left\|s_{n}^{*} s_{n}-a_{n}\right\|=0, \lim _{n \rightarrow \omega}\left\|b_{n} s_{n}-s_{n}\right\|=0 .
$$

(2) Let $S$ be a subset of $T_{1}(A)$. We say that $A$ has strict comparison (respectively, strict comparison with respect to $S$ ) if for any $k \in \mathbb{N}, a, b \in M_{k}(A)_{+}$with $d_{\tau \otimes \operatorname{Tr}_{k}}(a)<d_{\tau \otimes \operatorname{Tr}_{\mathrm{k}}}(b)$ for any $\tau \in T_{1}(A)$ (respectively, for any $\tau \in S$ ) implies $a \precsim b$.

Note that strict comparison in the definition above is different from almost unperforation of the Cuntz semigroup $\mathrm{Cu}(A)$. Essentially the same proofs as 39 , Lemma 4.7] and [39, Proposition 4.8] show the following theorem. See also [40, Propostion 3.3] and [2, Theorem 4.1].

Theorem 2.6. (Matui-Sato)

Let $A$ be a simple separable infinite-dimensional nuclear $\mathrm{C}^{*}$-algebra with finitely many extremal tracial states and no unbounded traces. Assume that $A$ has property (SI). Then:

(i) For any tracial state $\sigma$ on $F(A)$, there exists a tracial state $\tau$ on $A$ such that $\sigma=\tau_{\omega}$.

(ii) If $a$ and $b$ are positive elements in $F(A)$ satisfying $d_{\tau_{\omega}}(a)<d_{\tau_{\omega}}(b)$ for any $\tau \in T_{1}(A)$, then there exists an element $r \in F(A)$ such that $r^{*} b r=a$. Moreover, $F(A)$ has strict comparison.

2.4. Razak-Jacelon algebra. Let $\mathcal{W}$ be the Razak-Jacelon algebra studied in [21], which has trivial $K$-groups and a unique tracial state $\tau$ and no unbounded traces. The Razak-Jacelon algebra $\mathcal{W}$ is constructed as an inductive limit $\mathrm{C}^{*}$-algebra of Razak's building block in [46, that is,

$A(n, m)=\left\{f \in C([0,1]) \otimes M_{m}(\mathbb{C}) \mid f(0)=\operatorname{diag}(\overbrace{c, . ., c}^{k}, 0_{n}), f(1)=\operatorname{diag}(\overbrace{c, . ., c}^{k+1}), \quad\right.$,

where $n$ and $m$ are natural numbers with $n \mid m$ and $k:=m / n-1$. Let $\mathcal{O}_{2}$ denote the Cuntz algebra generated by 2 isometries $S_{1}$ and $S_{2}$. For every $\lambda_{1}, \lambda_{2} \in \mathbb{R}$ there exists by universality a one-parameter automorphism group $\alpha$ of $\mathcal{O}_{2}$ given by $\alpha_{t}\left(S_{j}\right)=e^{i t \lambda_{j}} S_{j}$. Kishimoto and Kumjian showed that if $\lambda_{1}$ and $\lambda_{2}$ are all non-zero, of the same sign and $\lambda_{1}$ and $\lambda_{2}$ generate $\mathbb{R}$ as a closed subgroup, then $\mathcal{O}_{2} \rtimes_{\alpha} \mathbb{R}$ is a simple stably projectionless $\mathrm{C}^{*}$-algebra with unique (up to scalar multiple) densely defined lower semicontinuous trace in [30] and [31. Moreover, Robert [4]. showed that $\mathcal{W} \otimes \mathbb{K}$ is isomorphic to $\mathcal{O}_{2} \rtimes_{\alpha} \mathbb{R}$ for some $\lambda_{1}$ and $\lambda_{2}$. (See also [9].)

By the uniqueness of traces on $\mathcal{W} \otimes \mathbb{K}$, for any automorphism $\alpha$ of $\mathcal{W} \otimes \mathbb{K}$, there exists a positive real number $\lambda(\alpha)$ such that $(\tau \otimes \operatorname{Tr}) \circ \alpha=\lambda(\alpha) \tau \otimes \operatorname{Tr}$. We say that $\alpha$ is a trace scaling automorphism if $\lambda(\alpha) \neq 1$. The following theorem is an immediate consequence of Razak's classification theorem (see also [47]).

Theorem 2.7. (Razak)

(i) Let $A$ be a simple unital approximately finite-dimensional (AF) algebra with a 
unique tracial state. Then $A \otimes \mathcal{W}$ is isomorphic to $\mathcal{W}$.

(ii) Every automorphism of $\mathcal{W}$ is approximately inner.

(iii) Let $\alpha$ and $\beta$ be automorphisms of $\mathcal{W} \otimes \mathbb{K}$. Then $\alpha$ and $\beta$ are approximately unitarily equivalent if and only if $\lambda(\alpha)=\lambda(\beta)$.

(iv) For any $\lambda \in \mathbb{R}_{+}^{\times}$, there exists an automorphism $\alpha$ of $\mathcal{W} \otimes \mathbb{K}$ such that $\lambda(\alpha)=\lambda$.

Note that $\mathcal{W}$ is UHF-stable by (i) in the theorem above, and hence $\mathcal{W}$ is $\mathcal{Z}$-stable. Hence $\mathcal{W}$ has strict comparison and property (SI) (see [50, 38] and [43]).

\section{Stable Uniqueness theorem}

In this section we shall recall some results in 12 and reformulate for our purpose. Let $\Omega$ be a compact metrizable space. The following proposition is based on the results of [7], 8], [1] and [14].

Proposition 3.1. (cf. [12, Proposition 8.2])

Let $A$ be a separable non-unital $\mathrm{C}^{*}$-algebra and $B$ a separable $\mathrm{C}^{*}$-algebra, and let $\sigma$ be a full homomorphism from $A$ to $B$. Suppose that $\varphi$ and $\psi$ are nuclear homomorphisms from $C(\Omega) \otimes A$ to $B$ with $[\varphi]=[\psi]$ in $K K_{\text {nuc }}(C(\Omega) \otimes A, B)$. Then for any finite subsets $F_{1} \subset C(\Omega), F_{2} \subset A$ and $\varepsilon>0$, there exist $m \in \mathbb{N}$, $z_{1}, z_{2}, \ldots, z_{m} \in \Omega$ and a unitary element $u$ in $M_{m^{2}+1}(B)^{\sim}$ such that

$$
\begin{aligned}
\| u^{*}(\varphi(f \otimes a) \oplus \overbrace{\left.\bigoplus_{k=1}^{m} f\left(z_{k}\right) \sigma(a) \oplus \cdots \oplus \bigoplus_{k=1}^{m} f\left(z_{k}\right) \sigma(a)\right) u}^{m} & \overbrace{\bigoplus_{\bigoplus_{k=1}^{m} f\left(z_{k}\right) \sigma(a) \oplus \cdots \oplus \bigoplus_{k=1}^{m} f\left(z_{k}\right) \sigma(a)}^{m} \|<\varepsilon}^{m} \\
& -\psi(f \otimes a)
\end{aligned}
$$

for any $f \in F_{1}$ and $a \in F_{2}$.

Proof. Choose a dense subset $\left\{z_{k} \mid k \in \mathbb{N}\right\} \subset \Omega$. Let $\gamma$ be a homomorphism from $C(\Omega) \otimes A$ to $M(B \otimes \mathbb{K} \otimes \mathbb{K})$ such that

$$
\gamma(f \otimes a)=\sigma(a) \otimes \sum_{k=1}^{\infty} f\left(z_{k}\right) e_{k k} \otimes 1
$$

for any $f \in C(\Omega)$ and $a \in A$, where $\left\{e_{i j}\right\}_{i, j \in \mathbb{N}}$ is the standard matrix units of $\mathbb{K}$. Then we see that $\gamma$ is purely large as an extension by [11, Theorem 17 (iii)]. The same arguments as in the proofs of [12, Lemma 8.1] and [12, Proposition 8.2] show that there exists a sequence $\left\{u_{n}\right\}_{n \in \mathbb{N}}$ of unitaries in $(B \otimes \mathbb{K})^{\sim}$ such that

$$
\left\|u_{n}(\varphi(f \otimes a) \oplus \gamma(f \otimes a)) u_{n}^{*}-\psi(f \otimes a) \oplus \gamma(f \otimes a)\right\| \rightarrow 0
$$

as $n \rightarrow \infty$ for any $f \in C(\Omega)$ and $a \in A$. For any $m \in \mathbb{N}$, let

$$
e_{m}:=1 \oplus \sum_{k=1}^{m} e_{k k} \otimes \sum_{k=1}^{m} e_{k k} \in B^{\sim} \oplus B^{\sim} \otimes \mathbb{K} \otimes \mathbb{K} \subset B^{\sim} \otimes \mathbb{K} .
$$

For any $n \in \mathbb{N}$, we have $\left\|\left[e_{m}, u_{n}\right]\right\| \rightarrow 0$ as $m \rightarrow \infty$. Hence for sufficiently large $m$, $e_{m} u_{n} e_{m}$ is close to a unitary element in $e_{m}(B \otimes \mathbb{K}) \sim e_{m} \cong M_{m^{2}+1}(B)^{\sim}$.

Therefore choose a sufficient large $n \in \mathbb{N}$, and then choose a sufficiently large $m \in \mathbb{N}$, we can find a unitary element $u$ in $M_{m^{2}+1}(B)^{\sim}$ satisfying the conclusion of the proposition.

In order to obtain a stable uniqueness theorem for $(G, \delta)$-multiplicative maps, we need to consider homomorphisms from $A$ to $\prod B_{n} / \bigoplus B_{n}$ for some $\mathrm{C}^{*}$-algebras $A$ and $B_{n}$. We can avoid the assumption of separability in the proposition above by 
Blackadar's technique (see [1, II.8.5] and [12, Lemma 8.4]). It is useful to consider the following for the fullness.

Definition 3.2. (cf. [12, Definition 8.7])

Let $L$ be a map from $A_{+, 1} \backslash\{0\} \times(0,1)$ to $\mathbb{N}$ and $N$ a map from $A_{+, 1} \backslash\{0\} \times(0,1)$ to $(0, \infty)$. A homomorphism $\varphi$ from $A$ to $B$ is said to be $(L, N)$-full if for any $\varepsilon \in(0,1), a \in A_{+, 1} \backslash\{0\}$ and $b \in B_{+, 1}$, there exist elements $x_{1}, x_{2}, \ldots, x_{L(a, \varepsilon)}$ in $B$ such that

$$
\left\|x_{i}\right\| \leq N(a, \varepsilon)
$$

for any $i=1,2, \ldots, L(a, \varepsilon)$ and

$$
\left\|b-\sum_{i=1}^{L(a, \varepsilon)} x_{i} \varphi(a) x_{i}^{*}\right\|<\varepsilon .
$$

The following proposition is a variant of [12, Proposition 8.12]. For finite sets $F_{1}$ and $F_{2}$, let $F_{1} \odot F_{2}:=\left\{a \otimes b \mid a \in F_{1}, b \in F_{2}\right\}$.

Proposition 3.3. Let $A$ be a separable non-unital nuclear $\mathrm{C}^{*}$-algebra that is $K K$ equivalent to $\{0\}$, and let $L: A_{+, 1} \backslash\{0\} \times(0,1) \rightarrow \mathbb{N}$ and $N: A_{+, 1} \backslash\{0\} \times(0,1) \rightarrow$ $(0, \infty)$ be maps. Then for any finite subsets $F_{1} \subset C(\Omega), F_{2} \subset A$ and $\varepsilon>0$, there exist finite subsets $G_{1} \subset C(\Omega), G_{2} \subset A, m \in \mathbb{N}$, and $\delta>0$ such that the following holds. Let $B$ be a $\mathrm{C}^{*}$-algebra. For any contractive $\left(G_{1} \odot G_{2}, \delta\right)$-multiplicative maps $\varphi, \psi: C(\Omega) \otimes A \rightarrow B$ and an $(L, N)$-full homomorphism $\sigma: A \rightarrow B$, there exist a unitary element in $u$ in $M_{m^{2}+1}(B)^{\sim}$ and $z_{1}, z_{2}, \ldots, z_{m} \in \Omega$ such that

$$
\begin{aligned}
\| u^{*}(\varphi(f \otimes a) \oplus & \overbrace{\left.\bigoplus_{k=1}^{m} f\left(z_{k}\right) \sigma(a) \oplus \cdots \oplus \bigoplus_{k=1}^{m} f\left(z_{k}\right) \sigma(a)\right) u}^{m} \\
& -\psi(f \otimes a) \oplus \overbrace{\bigoplus_{k=1}^{m} f\left(z_{k}\right) \sigma(a) \oplus \cdots \oplus \bigoplus_{k=1}^{m} f\left(z_{k}\right) \sigma(a)}^{m} \|<\varepsilon
\end{aligned}
$$

for any $f \in F_{1}$ and $a \in F_{2}$.

Proof. Let finite subsets $F_{1} \subset C(\Omega), F_{2} \subset A$ and $\varepsilon>0$. On the contrary, suppose that the proposition were false for $F_{1}, F_{2}$ and $\varepsilon$. Then for any $n \in \mathbb{N}$, there exist a $\mathrm{C}^{*}$-algebra $B_{n}$, contractive c.p. maps $\varphi_{n}, \psi_{n}: C(\Omega) \otimes A \rightarrow B_{n}$ and an $(L, N)$-full homomorphism $\sigma_{n}: A \rightarrow B_{n}$ such that

$$
\left\|\varphi_{n}(x y)-\varphi_{n}(x) \varphi_{n}(y)\right\| \rightarrow 0, \quad\left\|\psi_{n}(x y)-\psi_{n}(x) \psi_{n}(y)\right\| \rightarrow 0
$$

as $n \rightarrow \infty$ for any $x, y \in C(\Omega) \otimes A$ and there exist no unitaries in $M_{n^{2}+1}\left(B_{n}\right)^{\sim}$ and $n$ elements in $\Omega$ satisfying the conclusion of the proposition.

Define homomorphisms $\Phi$ and $\Psi$ from $C(\Omega) \otimes A$ to $\prod B_{n} / \oplus B_{n}$ by

$$
\Phi(x):=\left(\varphi_{n}(x)\right)_{n}, \quad \Psi(x):=\left(\psi_{n}(x)\right)_{n}
$$

for any $x \in C(\Omega) \otimes A$, and define a homomorphism $\Sigma$ from $A$ to $\prod B_{n} / \bigoplus B_{n}$ by

$$
\Sigma(a):=\left(\sigma_{n}(a)\right)_{n}
$$

for any $a \in A$. Since $\sigma_{n}$ is $(L, N)$-full for any $n \in \mathbb{N}, \Sigma$ is full in $\prod B_{n} / \bigoplus B_{n}$. By [12, Lemma 8.4], there exists a separable $\mathrm{C}^{*}$-subalgebra $B$ of $\prod B_{n} / \bigoplus B_{n}$ such that

$$
\Phi(C(\Omega) \otimes A), \Psi(C(\Omega) \otimes A), \Sigma(A) \subset B
$$

and $\Sigma$ is full in $B$. 
Since $C(\Omega) \otimes A$ is non-unital, nuclear and $K K$-equivalent to $\{0\}$, it follows from Proposition 3.1 that there exist $m \in \mathbb{N}, z_{1}, z_{2}, \ldots, z_{m} \in \Omega$ and a unitary element $U$ in $M_{m^{2}+1}(B)^{\sim}$ such that

$$
\begin{aligned}
\| U^{*}(\Phi(f \otimes a) \oplus \overbrace{\left.\bigoplus_{k=1}^{m} f\left(z_{k}\right) \Sigma(a) \oplus \cdots \oplus \bigoplus_{k=1}^{m} f\left(z_{k}\right) \Sigma(a)\right)} & \overbrace{\bigoplus_{\bigoplus_{k=1}^{m} f\left(z_{k}\right) \Sigma(a) \oplus \cdots \oplus \bigoplus_{k=1}^{m} f\left(z_{k}\right) \Sigma(a)}^{m} \|<\varepsilon}^{m} \\
& -\Psi(f \otimes a)
\end{aligned}
$$

for any $f \in F_{1}$ and $a \in F_{2}$. It is easy to see that $U$ can be lifted to a unitary element $\left(u_{n}\right)_{n \in \mathbb{N}}$ in $\prod M_{m^{2}+1}\left(B_{n}\right)^{\sim}$. Note that we may assume $u_{n}=b_{n}+1$ for some $b_{n} \in M_{m^{2}+1}\left(B_{n}\right)$. For sufficiently large $n \in \mathbb{N}$, we have

$$
\begin{aligned}
\| u_{n}^{*}(\varphi_{n}(f \otimes a) \oplus \overbrace{\left.\bigoplus_{k=1}^{m} f\left(z_{k}\right) \sigma_{n}(a) \oplus \cdots \oplus \bigoplus_{k=1}^{m} f\left(z_{k}\right) \sigma_{n}(a)\right)}^{m} & \overbrace{\bigoplus_{\bigoplus}^{m} f\left(z_{k}\right) \sigma_{n}(a) \oplus \cdots \oplus \bigoplus_{k=1}^{m} f\left(z_{k}\right) \sigma_{n}(a)}^{m} \|<\varepsilon \\
& -\psi_{n}(f \otimes a) \oplus \underbrace{m}_{k=1}
\end{aligned}
$$

for any $f \in F_{1}$ and $a \in F_{2}$. Take elements $z_{m+1}, \ldots, z_{n} \in \Omega$, and let $u$ be a trivial unitary extension in $M_{n^{2}+1}\left(B_{n}\right) \sim$ from $u_{n}$. Then

$$
\begin{aligned}
\| u^{*}(\varphi_{n}(f \otimes a) \oplus \overbrace{\left.\bigoplus_{k=1}^{n} f\left(z_{k}\right) \sigma_{n}(a) \oplus \cdots \oplus \bigoplus_{k=1}^{n} f\left(z_{k}\right) \sigma_{n}(a)\right)} u \\
\\
-\psi_{n}(f \otimes a) \oplus \overbrace{\bigoplus_{k=1}^{n} f\left(z_{k}\right) \sigma_{n}(a) \oplus \cdots \oplus \bigoplus_{k=1}^{n} f\left(z_{k}\right) \sigma_{n}(a)}^{n} \|<\varepsilon
\end{aligned}
$$

for any $f \in F_{1}$ and $a \in F_{2}$. This is a contradiction. Therefore the proof is complete.

The following lemma is an analogous lemma of [23, Lemma 2.2 (iii)].

Lemma 3.4. Let $A$ be a $C^{*}$-algebra, and let $L: A_{+, 1} \backslash\{0\} \times(0,1) \rightarrow \mathbb{N}$ and $N: A_{+, 1} \backslash\{0\} \times(0,1) \rightarrow(0, \infty)$ be maps. Then there exist maps $L^{\prime}: A_{+, 1} \backslash$ $\{0\} \times(0,1) \rightarrow \mathbb{N}$ and $N^{\prime}: A_{+, 1} \backslash\{0\} \times(0,1) \rightarrow(0, \infty)$ such that the following holds. Let $B$ be a $C^{*}$-algebra, and let $C$ be a hereditary subalgebra of $B$. If $\sigma$ is a homomorphism from $A$ to $C \subset B$ such that for any $\varepsilon \in(0,1), a \in A_{+, 1} \backslash\{0\}$ and $b \in C_{+, 1}$, there exist elements $x_{1}, x_{2}, \ldots, x_{L(a, \varepsilon)}$ in $B$ such that

$$
\left\|x_{i}\right\| \leq N(a, \varepsilon)
$$

for any $i=1,2, \ldots, L(a, \varepsilon)$ and

$$
\left\|b-\sum_{i=1}^{L(a, \varepsilon)} x_{i} \sigma(a) x_{i}^{*}\right\|<\varepsilon,
$$

then $\sigma$ is $\left(L^{\prime}, N^{\prime}\right)$-full in $C$.

Proof. For any $a \in A_{+, 1} \backslash\{0\}$ and $\varepsilon \in(0,1)$, let

$$
L^{\prime}(a, \varepsilon):=L\left(a^{2}, \varepsilon\right), \quad N^{\prime}(a, \varepsilon):=N\left(a^{2}, \varepsilon\right) .
$$


Then $L^{\prime}$ and $N^{\prime}$ have the desired property. Indeed, let $\sigma$ be a homomorphism from $A$ to $C \subset B$ satisfying the assumption above. For any $b \in C_{+, 1}$ and $\varepsilon \in(0,1)$, there exist elements $x_{1}, x_{2}, \ldots, x_{L^{\prime}(a, \varepsilon)}$ in $B$ such that $\left\|x_{i}\right\| \leq N^{\prime}(a, \varepsilon)$ for any $i=$ $1,2, \ldots, L^{\prime}(a, \varepsilon)$ and

$$
\left\|b^{\frac{1}{2}}-\sum_{i=1}^{L^{\prime}(a, \varepsilon)} x_{i} \sigma\left(a^{2}\right) x_{i}^{*}\right\|<\varepsilon
$$

We have

$$
\left\|b-\sum_{i=1}^{L^{\prime}(a, \varepsilon)} b^{\frac{1}{4}} x_{i} \sigma\left(a^{\frac{1}{2}}\right) \sigma(a) \sigma\left(a^{\frac{1}{2}}\right) x_{i}^{*} b^{\frac{1}{4}}\right\| \leq\left\|b^{\frac{1}{2}}-\sum_{i=1}^{L^{\prime}(a, \varepsilon)} x_{i} \sigma\left(a^{2}\right) x_{i}^{*}\right\|<\varepsilon .
$$

Since $C$ is a hereditary subalgebra of $B$, we see that $b^{\frac{1}{4}} x_{i} \sigma\left(a^{\frac{1}{2}}\right) \in C$ for any $i=$ $1,2, \ldots, L^{\prime}(a, \varepsilon)$. Therefore $\sigma$ is $\left(L^{\prime}, N^{\prime}\right)$-full in $C$.

Essentially the same proof as [12, Lemma 8.15] show the following lemma. Roughly speaking, this lemma says that if target algebras have strict comparison, then the $(L, N)$-fullness can be controlled by traces.

Lemma 3.5. (Elliott-Niu)

For any $\varepsilon>0$ and $\delta>0$, there exist $\ell(\delta) \in \mathbb{N}$ and $n(\varepsilon)>0$ such that the following holds. Let $A$ be a $\mathrm{C}^{*}$-algebra, and let $S$ be a subset of $T_{1}(A)$. Assume that $A$ has strict comparison with respect to $S$. If $a$ and $b$ are positive contractions in $A$ such that

$$
\tau(a)>d_{\tau}(b) \delta
$$

for any $\tau \in S$, then there exist $x_{1}, x_{2}, \ldots, x_{\ell(\delta)} \in A$ such that

$$
\left\|x_{i}\right\| \leq n(\varepsilon)
$$

for any $i=1,2, \ldots, \ell(\delta)$ and

$$
\left\|b-\sum_{i=1}^{\ell(\delta)} x_{i} a x_{i}^{*}\right\|<\varepsilon .
$$

The following lemma is a variant of [35, Lemma 3.5].

Lemma 3.6. Let $A$ be $\sigma$-unital $\mathrm{C}^{*}$-algebra, and let $\tau$ be an extremal tracial state on $A$. If $a$ is a positive element in $F(A)$, then

$$
\tau_{\omega}(\rho(a \otimes x))=\tau_{\omega}(a) \tau(x)
$$

for any $x \in A$.

Proof. There exists a positive contraction $\left(a_{n}\right)_{n}$ in $\mathcal{W}_{\omega}$ such that $a=\left[\left(a_{n}\right)_{n}\right]$. Note that we have $\rho(a \otimes x)=\left(a_{n} x\right)_{n}=\left(a_{n}^{\frac{1}{2}} x a_{n}^{\frac{1}{2}}\right)_{n}$. For any $x \in A$, define $\tau^{\prime}(x):=$ $\lim _{n \rightarrow \omega} \tau\left(a_{n} x\right)$. Then $\tau^{\prime}$ is a trace on $A$ and $\tau^{\prime} \leq \tau$. Since $\tau$ is an extremal tracial state on $A$, there exists $\lambda \geq 0$ such that $\tau^{\prime}=\lambda \tau$. Let $\left\{h_{m}\right\}_{m \in \mathbb{N}}$ be an approximate unit for $A$. Then $\lim _{m \rightarrow \infty} \tau\left(h_{m}\right)=1$ because $\tau$ is a state. Hence $\lambda=\lim _{m \rightarrow \infty} \tau^{\prime}\left(h_{m}\right)$. Similar arguments as in the proof of [43, Proposition 5.3] show

$$
\lim _{m \rightarrow \infty} \lim _{n \rightarrow \omega} \tau\left(a_{n} h_{m}\right)=\lim _{n \rightarrow \omega} \tau\left(a_{n}\right) .
$$

Therefore $\lambda=\lim _{n \rightarrow \omega} \tau\left(a_{n}\right)$. We obtain the conclusion.

For a projection $p$ in $F(\mathcal{W})$, define a homomorphism $\sigma_{p}$ from $\mathcal{W}$ to $\mathcal{W}_{p}^{\omega}$ by

$$
\sigma_{p}(x):=\rho(p \otimes x)
$$

for any $x \in \mathcal{W}$. 
Proposition 3.7. There exist maps $L: \mathcal{W}_{+, 1} \backslash\{0\} \times(0,1) \rightarrow \mathbb{N}$ and $N: \mathcal{W}_{+, 1} \backslash$ $\{0\} \times(0,1) \rightarrow(0, \infty)$ such that the following holds. If $p$ be a projection in $F(\mathcal{W})$ such that $\tau_{\omega}(p)>0$ where $\tau$ is the unique tracial state on $\mathcal{W}$, then $\sigma_{p}$ is $(L, N)$-full.

Proof. For any $\varepsilon>0$ and $\delta>0$, take $\ell(\delta) \in \mathbb{N}$ and $n(\varepsilon)>0$ in Lemma 3.5. Define

$$
L(a, \varepsilon):=\ell\left(\frac{\tau(a)}{2}\right), \quad N(a, \varepsilon):=n(\varepsilon)
$$

for any $a \in \mathcal{W}_{+, 1} \backslash\{0\}$ and $\varepsilon \in(0,1)$. Note that $\tau(a)>0$ since $\mathcal{W}$ is simple.

Let $p \in F(\mathcal{W})$ be a projection in $F(\mathcal{W})$ such that $\tau_{\omega}(p)>0$. There exists a positive contraction $\left(p_{n}\right)_{n}$ in $\mathcal{W}_{\omega}$ such that $p=\left[\left(p_{n}\right)_{n}\right]$. Let $a \in \mathcal{W}_{+, 1} \backslash\{0\}$, $b \in\left(\mathcal{W}_{p}^{\omega}\right)_{+, 1}$ and $\varepsilon>0$. Since $\mathcal{W}_{p}^{\omega}=\overline{\rho(p \otimes s) \mathcal{W}^{\omega} \rho(p \otimes s)}=\overline{\left(p_{n} s\right)_{n} \mathcal{W}^{\omega}\left(s p_{n}\right)_{n}}$ where $s$ is a strictly positive element in $\mathcal{W}$, we have $\left(p_{n}\right)_{n} b\left(p_{n}\right)_{n}=b$. Hence $d_{\tau_{\omega}}(b) \leq \tau_{\omega}(p)$. By Lemma 3.6 and $\tau_{\omega}(p)>0$, we have

$$
\tau_{\omega}\left(\sigma_{p}(a)\right)=\tau_{\omega}(p) \tau(a)>\tau_{\omega}(p) \frac{\tau(a)}{2} \geq d_{\tau_{\omega}}(b) \frac{\tau(a)}{2} .
$$

Since $\mathcal{W}$ has strict comparison and the tracial state on $\mathcal{W}$ is unique, $\mathcal{W}^{\omega}$ has strict comparison with respect to $\left\{\tau_{\omega}\right\}$ (see, for example, the proof of [2, Lemma 1.23]). Therefore Lemma 3.5 implies that there exist elements $x_{1}, x_{2}, \ldots, x_{L(a, \varepsilon)}$ in $\mathcal{W}^{\omega}$ such that

$$
\left\|x_{i}\right\| \leq N(a, \varepsilon)
$$

for any $i=1,2, \ldots, L(a, \varepsilon)$ and

$$
\left\|b-\sum_{i=1}^{L(a, \varepsilon)} x_{i} \sigma_{p}(a) x_{i}^{*}\right\|<\varepsilon .
$$

Since $\mathcal{W}_{p}^{\omega}$ is a hereditary subalgebra of $\mathcal{W}^{\omega}$, we obtain the conclusion by Lemma 3.4

The following corollary is an immediate consequence of Proposition 3.3 and Proposition 3.7 .

Corollary 3.8. For any finite subsets $F_{1} \subset C(\Omega), F_{2} \subset \mathcal{W}, \varepsilon>0$, there exist finite subsets $G_{1} \subset C(\Omega), G_{2} \subset \mathcal{W}, m \in \mathbb{N}$ and $\delta>0$ such that the following holds. Let $p$ be a projection in $F(\mathcal{W})$ such that $\tau_{\omega}(p)>0$ where $\tau$ is the unique tracial state on $\mathcal{W}$. For any contractive $\left(G_{1} \odot G_{2}, \delta\right)$-multiplicative maps $\varphi, \psi: C(\Omega) \otimes \mathcal{W} \rightarrow \mathcal{W}_{p}^{\omega}$, there exist a unitary element $u$ in $M_{m^{2}+1}\left(\mathcal{W}_{p}^{\omega}\right)^{\sim}$ and $z_{1}, z_{2}, \ldots, z_{m} \in \Omega$ such that

$$
\begin{aligned}
\| u^{*}(\varphi(f \otimes a) \oplus \overbrace{\left.\bigoplus_{k=1}^{m} f\left(z_{k}\right) \rho(p \otimes a) \oplus \cdots \oplus \bigoplus_{k=1}^{m} f\left(z_{k}\right) \rho(p \otimes a)\right)} & \overbrace{\overbrace{\bigoplus_{k=1}^{m} f\left(z_{k}\right) \rho(p \otimes a) \oplus \cdots \oplus \bigoplus_{k=1}^{m} f\left(z_{k}\right) \rho(p \otimes a)}^{m} \|<\varepsilon}^{m} \\
& -\psi(f \otimes a)
\end{aligned}
$$

for any $f \in F_{1}$ and $a \in F_{2}$.

\section{Properties of $F(\mathcal{W})$}

In this section we shall consider properties of $F(\mathcal{W})$. In the rest of this paper, we denote by $\tau$ the unique tracial state on $\mathcal{W}$. Since $\mathcal{W}$ has property (SI), the following proposition is an immediate consequence of Theorem 2.6 . 
Proposition 4.1. (i) The central sequence $\mathrm{C}^{*}$-algebra $F(\mathcal{W})$ has a unique tracial state $\tau_{\omega}$.

(ii) If $a$ and $b$ are positive elements in $F(\mathcal{W})$ satisfying $d_{\tau_{\omega}}(a)<d_{\tau_{\omega}}(b)$, then there exists an element $r \in F(\mathcal{W})$ such that $r^{*} b r=a$. Moreover, $F(\mathcal{W})$ has strict comparison.

The following proposition shows that $F(\mathcal{W})$ has many projections.

Proposition 4.2. (i) For any $N \in \mathbb{N}$, there exists a unital homomorphism from $M_{N}(\mathbb{C})$ to $F(\mathcal{W})$.

(ii) For any $\theta \in[0,1]$, there exists a non-zero projection $p$ in $F(\mathcal{W})$ such that $\tau_{\omega}(p)=\theta$.

(iii) Let $h$ be a positive element in $F(\mathcal{W})$ such that $d_{\tau_{\omega}}(h)>0$. For any $\theta \in$ $\left[0, d_{\tau_{\omega}}(h)\right)$, there exists a non-zero projection $p$ in $\overline{h F(\mathcal{W}) h}$ such that $\tau_{\omega}(p)=\theta$.

Proof. Let $\left\{h_{n}\right\}_{n \in \mathbb{N}}$ be an approximate unit for $\mathcal{W}$.

(i) By Theorem 2.7. $\mathcal{W}$ is isomorphic to $\mathcal{W} \otimes M_{N}=\mathcal{W} \otimes \bigotimes_{n \in \mathbb{N}} M_{N}(\mathbb{C})$. Define a map $\varphi$ from $M_{N}(\mathbb{C})$ to $F(\mathcal{W}) \cong F\left(\mathcal{W} \otimes \bigotimes_{n \in \mathbb{N}} M_{N}(\mathbb{C})\right)$ by

$$
\varphi(x):=\left[(h_{n} \otimes \overbrace{1 \otimes \cdots \otimes 1}^{n} \otimes x \otimes 1 \otimes \cdots)_{n}\right]
$$

for any $x \in M_{N}(\mathbb{C})$. Then $\varphi$ is a unital homomorphism.

(ii) Since $\mathbb{Z}[1 / 2]$ is dense in $\mathbb{R}$, there exists a sequence $\left\{q_{n}\right\}_{n \in \mathbb{N}}$ of non-zero projections in $M_{2 \infty}$ such that $\lim _{n \rightarrow \infty} \tau^{\prime}\left(q_{n}\right)=\theta$, where $\tau^{\prime}$ is the unique tracial state on $M_{2}$. Put

$$
p:=\left[(h_{n} \otimes \overbrace{1 \otimes \cdots \otimes 1}^{n} \otimes q_{n} \otimes 1 \otimes \cdots)_{n}\right] \in F\left(\mathcal{W} \otimes \bigotimes_{n \in \mathbb{N}} M_{2^{\infty}}\right) \cong F(\mathcal{W}) .
$$

Then $p$ is a non-zero projection in $F(\mathcal{W})$ such that $\tau_{\omega}(p)=\theta$.

(iii) There exists a non-zero projection $q$ in $F(\mathcal{W})$ such that $\tau_{\omega}(q)=\theta$ by (ii). Proposition 4.1 implies that there exists an element $r$ in $F(\mathcal{W})$ such that $r h r^{*}=q$. Let $p:=h^{\frac{1}{2}} r^{*} r h^{\frac{1}{2}}$, then $p$ is a non-zero projection in $\overline{h F(\mathcal{W}) h}$ such that $\tau_{\omega}(p)=$ $\theta$.

Recall that $\operatorname{ker}\left(\varrho_{\mathcal{W}}\right)=\left\{x \in F(\mathcal{W}) \mid \tau_{\omega}\left(x^{*} x\right)=0\right\}$.

Proposition 4.3. Let $x$ be an element in $F(\mathcal{W})$. Then $x$ is full if and only if $x \notin \operatorname{ker}(\varrho \mathcal{W})$.

Proof. It is obvious that if $x \in \operatorname{ker}(\varrho \mathcal{W})$, then $x$ is not full in $F(\mathcal{W})$. Let $x \notin$ $\operatorname{ker}\left(\varrho_{\mathcal{W}}\right)$, then $\tau_{\omega}\left(x^{*} x\right)>0$. For any $b \in F(\mathcal{W})_{+}$, there exists a positive number $\delta$ such that $\tau_{\omega}\left(x^{*} x\right)>\delta d_{\tau_{\omega}}(b)$. Hence, for any $\varepsilon>0$, there exist $z_{1}, z_{2}, \ldots, z_{\ell(\delta)} \in$ $F(\mathcal{W})$ such that

$$
\left\|b-\sum_{i=1}^{\ell(\delta)} z_{i} x^{*} x z_{i}^{*}\right\|<\varepsilon
$$

by Lemma 3.5. This shows the closed ideal generated by $x$ is equal to $F(\mathcal{W})$. Therefore $x$ is full.

Using the ideas in [49] and [48, we shall show that certain elements in $F(\mathcal{W})$ can be approximated by invertible elements. We denote by $\mathrm{GL}(A)$ the set of invertible elements in $A$. 
Lemma 4.4. Let $a$ and $b$ be positive elements in $F(\mathcal{W})$ such that $a, b \notin \operatorname{ker}(\varrho \mathcal{W})$. Then there exist a unitary element $u$ and a projection $p^{\prime}$ in $F(\mathcal{W})$ such that $p^{\prime} \notin$ $\operatorname{ker}(\varrho \mathcal{W})$ and $p^{\prime} \in \overline{a F(\mathcal{W}) a} \cap u(\overline{b F(\mathcal{W}) b}) u^{*}$

Proof. Since $a, b \notin \operatorname{ker}(\varrho \mathcal{W})$, we have $d_{\tau_{\omega}}(a)>0$ and $d_{\tau_{\omega}}(b)>0$. Proposition 4.2 and Proposition 4.1 imply that there exist a projection $p \in \overline{a F(\mathcal{W}) a}$ and an element $r \in F(\mathcal{W})$ such that $p \notin \operatorname{ker}(\varrho \mathcal{W})$ and $r b r^{*}=p$. Then we have $p r b \notin \operatorname{ker}(\varrho \mathcal{W})$, and hence $a r b \notin \operatorname{ker}(\varrho \mathcal{W})$. Since $r$ is a linear combination of four unitaries in $F(\mathcal{W})$, there exists a unitary element $w$ in $F(\mathcal{W})$ such that $a w b \notin \operatorname{ker}(\varrho \mathcal{W})$. Since $\operatorname{ker}(\varrho \mathcal{W})$ is closed, essentially the same argument as in the proof of [49, Lemma 3.4] show that there exist a unitary element $u$ and a positive element $c \in F(\mathcal{W})$ such that $c \notin \operatorname{ker}(\varrho \mathcal{W})$ and $c \in \overline{a F(\mathcal{W}) a} \cap u(\overline{b F(\mathcal{W}) b}) u^{*}$. Using Proposition 4.2 we can find a projection $p^{\prime}$ satisfying the conclusion of the proposition.

Lemma 4.5. Let $x$ be an element in $F(\mathcal{W})$. Assume that there exist projections $p$ and $q$ in $F(\mathcal{W})$ such that $x p=q x=x$ and $1-p, 1-q \notin \operatorname{ker}(\varrho \mathcal{W})$. Then there exist a unitary element $u$ and a projection $e$ in $F(\mathcal{W})$ such that $e u x=u x e=u x$ and $1-e \notin \operatorname{ker}(\varrho \mathcal{W})$.

Proof. Lemma 4.4 implies that there exist a unitary element $u$ and a projection $p^{\prime}$ in $F(\mathcal{W})$ such that $p^{\prime} \notin \operatorname{ker}(\varrho \mathcal{W})$ and

$$
p^{\prime} \in(1-p) F(\mathcal{W})(1-p) \cap u(1-q) F(\mathcal{W})(1-q) u^{*} .
$$

Since $x(1-p)=(1-q) x=0$, we have $u x p^{\prime}=0$ and $p^{\prime} u x=0$. Put $e:=1-p^{\prime}$, then we obtain the conclusion.

Lemma 4.6. Let $y$ be an element in $F(\mathcal{W})$. Assume that there exists a projection $e$ in $F(\mathcal{W})$ such that $e y=y e=y$ and $1-e \notin \operatorname{ker}(\varrho \mathcal{W})$. Then $y \in \overline{\operatorname{GL}(F(\mathcal{W}))}$.

Proof. It is enough to show that $y$ is a product of two nilpotent elements. Since $1-e$ is full by Proposition 4.3, there exist elements $x_{1}, x_{2}, \ldots, x_{N}$ in $F(\mathcal{W})$ such that $\sum_{i=1}^{N} x_{i}(1-e) x_{i}^{*}=1$. Proposition 4.2 implies that there exists a unital homomorphism $\varphi$ from $M_{N+1}(\mathbb{C})$ to $F(\mathcal{W})$. Let $\left\{e_{i j}\right\}_{i, j=1}^{N+1}$ be the standard matrix units of $M_{N+1}(\mathbb{C})$. Taking suitable subsequences of representatives of $\varphi\left(e_{i j}\right)$ for any $i, j=1,2, \ldots, N+1$, we may assume that the range of $\varphi$ commutes with $y, e$, $x_{1}, \ldots, x_{N}$. Put

$$
r_{1}:=\sum_{i=1}^{N} e x_{i}(1-e) \varphi\left(e_{N+1 i}\right), \quad r_{2}:=\sum_{i=1}^{N} y \varphi\left(e_{i i+1}\right), \quad r:=r_{1}+r_{2}
$$

and

$$
t_{1}:=\sum_{i=1}^{N}(1-e) x_{i}^{*} y \varphi\left(e_{i N+1}\right), \quad t_{2}:=\sum_{i=1}^{N} e \varphi\left(e_{i+1 i}\right), \quad t:=t_{1}+t_{2} .
$$

Then similar arguments as in the proof of [48, Lemma 2.1] show that $r t=y$ and $r^{N+2}=t^{N+2}=0$. Indeed, we have

$r t=\left(r_{1}+r_{2}\right)\left(t_{1}+t_{2}\right)=r_{1} t_{1}+r_{2} t_{2}=\sum_{i=1}^{N} e x_{i}(1-e) x_{i}^{*} y \varphi\left(e_{N+1 N+1}\right)+\sum_{i=1}^{N} y \varphi\left(e_{i i}\right)=y$.

Since we have $r_{1} r_{2}=0, r^{k}=\left(r_{1}+r_{2}\right)^{k}=\sum_{i=0}^{k} r_{2}^{i} r_{1}^{k-i}$ for any $k \in \mathbb{N}$. It can be easily checked that $r_{1}^{2}=0$ and $r_{2}^{N+1}=0$. This implies that $r^{N+2}=0$. In a similar way, we see that $t^{N+2}=0$.

The following proposition is an immediate consequence of Lemma 4.5] and Lemma 4.6 
Proposition 4.7. Let $x$ be an element in $F(\mathcal{W})$. Assume that there exist projections $p$ and $q$ in $F(\mathcal{W})$ such that $x p=q x=x$ and $1-p, 1-q \notin \operatorname{ker}(\varrho \mathcal{W})$. Then $x \in \overline{\mathrm{GL}(F(\mathcal{W}))}$.

Using the proposition above, we shall show that for certain projections in $F(\mathcal{W})$, Murray-von Neumann equivalence and unitary equivalence coincide.

Proposition 4.8. Let $p$ and $q$ be projections in $F(\mathcal{W})$ such that $\tau_{\omega}(p)<1$. Then $p$ and $q$ are Murray-von Neumann equivalent if and only if $p$ and $q$ are unitarily equivalent.

Proof. The if part is obvious. We will show the only if part. Suppose that there exists a partial isometry $v \in F(\mathcal{W})$ such that $v^{*} v=p$ and $v v^{*}=q$. Since we have $v p=q v=v$ and $\tau_{\omega}(1-q)=\tau_{\omega}(1-p)>0$, there exists an invertible element $s$ of norm one such that $\|s-v\|<1 / 4$ by Proposition 4.7 Let $u:=s\left(s^{*} s\right)^{-\frac{1}{2}}$. Then $u$ is a unitary element in $F(\mathcal{W})$ and we have $\left\|u p u^{*}-q\right\|<1$. Therefore we see that $p$ is unitarily equivalent to $q$.

We shall show that every unitary element in $F(\mathcal{W})$ can be lifted to a unitary element in $\mathcal{W}_{\omega}^{\sim}$.

Proposition 4.9. Let $A$ be a $\sigma$-unital $\mathrm{C}^{*}$-algebra with $A \subset \overline{\mathrm{GL}\left(A^{\sim}\right)}$. If $u$ is a unitary element in $F(A)$, then there exists a unitary element $w$ in $A_{\omega}^{\sim}$ such that $u=[w]$.

Proof. Since $A \subset \overline{\mathrm{GL}\left(A^{\sim}\right)}$, there exists a bounded sequence $\left\{z_{n}\right\}_{n \in \mathbb{N}}$ of invertible elements in $A^{\sim}$ such that $u=\left[\left(z_{n}\right)_{n}\right]$. Note that for any $a \in A$,

$$
\lim _{n \rightarrow \omega}\left\|z_{n}^{*} z_{n} a-a\right\|=0, \quad \lim _{n \rightarrow \omega}\left\|z_{n} z_{n}^{*} a-a\right\|=0
$$

because $u$ is a unitary element in $F(A)$. For any $n \in \mathbb{N}$, let $w_{n}:=z_{n}\left(z_{n}^{*} z_{n}\right)^{-\frac{1}{2}}$. Then $w_{n}$ is a unitary element in $A^{\sim}$ and for any $a \in A$, we have

$$
\left\|w_{n} a-z_{n} a\right\|=\left\|w_{n}\left(a-\left(z_{n}^{*} z_{n}\right)^{\frac{1}{2}} a\right)\right\|=\left\|a-\left(z_{n}^{*} z_{n}\right)^{\frac{1}{2}} a\right\| \rightarrow 0
$$

as $n \rightarrow \omega$. Furthermore, for any $a \in A$, we have

$$
\begin{aligned}
\left\|\left[w_{n}, a\right]\right\| & =\left\|w_{n} a w_{n}^{*}-a\right\| \\
& =\left\|w_{n} a w_{n}^{*}-z_{n} a w_{n}^{*}+z_{n} a w_{n}^{*}-z_{n} a z_{n}^{*}+z_{n} a z_{n}^{*}-z_{n} z_{n}^{*} a+z_{n} z_{n}^{*} a-a\right\| \\
& \leq\left\|w_{n} a-z_{n} a\right\|+\left\|z_{n}\right\|\left\|w_{n} a^{*}-z_{n} a^{*}\right\|+\left\|z_{n}\right\|\left\|a z_{n}^{*}-z_{n}^{*} a\right\|+\left\|z_{n} z_{n}^{*} a-a\right\| \\
& \rightarrow 0
\end{aligned}
$$

as $n \rightarrow \omega$. Therefore $\left(w_{n}\right)_{n}$ is a unitary element in $A_{\omega}^{\sim}$ such that $u=\left[\left(w_{n}\right)_{n}\right]$.

Since $\mathcal{W}$ has stable rank one, we have the following corollary.

Corollary 4.10. Let $u$ be a unitary element in $F(\mathcal{W})$. Then there exists a unitary element $w$ in $\mathcal{W}_{\omega}^{\sim}$ such that $u=[w]$.

\section{Homotopy of Unitaries in $F(\mathcal{W})$}

In this section we shall prove Theorem 5.7. The following lemma is motivated by [36, Lemma 4.1] and [36, Lemma 4.2].

Lemma 5.1. Let $\Omega$ be a compact metrizable space, and let $F$ be a finite subset of $C(\Omega)$ and $\varepsilon>0$. Suppose that $\varphi$ and $\psi$ are unital homomorphisms from $C(\Omega)$ to $F(\mathcal{W})$ such that $\tau_{\omega} \circ \varphi=\tau_{\omega} \circ \psi$. Then there exist a projection $p \in F(\mathcal{W})$, $(F, \varepsilon)$-multiplicative unital c.p. maps $\varphi^{\prime}$ and $\psi^{\prime}$ from $C(\Omega)$ to $p F(\mathcal{W}) p$ and a unital homomorphism $\sigma$ from $C(\Omega)$ to $(1-p) F(\mathcal{W})(1-p)$ with finite-dimensional range such that

$$
0<\tau_{\omega}(p)<\varepsilon, \quad \varphi \sim_{F, \varepsilon} \varphi^{\prime} \oplus \sigma, \quad \psi \sim_{F, \varepsilon} \psi^{\prime} \oplus \sigma .
$$


Proof. We may assume that every element in $F$ is of norm one. Let $\mu$ be the probability measure on $\Omega$ corresponding to $\tau_{\omega} \circ \varphi=\tau_{\omega} \circ \psi$. By the same argument as in the proof of [36. Lemma 4.1], there exist pairwise disjoint open subsets $W_{1}, W_{2}, \ldots, W_{l} \subset \Omega$ such that

$$
\mu\left(\Omega \backslash \bigcup_{i=1}^{l} W_{i}\right)=0, \quad \mu\left(W_{i}\right)>0
$$

and $|f(x)-f(y)|<\varepsilon / 3$ for any $x, y \in W_{i}$ and $f \in F$. For any $i=1,2, \ldots, l$, choose $z_{i} \in W_{i}$. Proposition 4.2 implies that there exists a projection $p_{i}$ in $\overline{\varphi\left(C_{0}\left(W_{i}\right)\right) F(\mathcal{W}) \varphi\left(C_{0}\left(W_{i}\right)\right)}$ such that

$$
\mu\left(W_{i}\right)-\frac{\varepsilon}{l}<\tau_{\omega}\left(p_{i}\right)<\mu\left(W_{i}\right) .
$$

Note that we have

$$
\left\|p_{i} \varphi(f)-f\left(z_{i}\right) p_{i}\right\|<\frac{\varepsilon}{3}, \quad\left\|\varphi(f) p_{i}-f\left(z_{i}\right) p_{i}\right\|<\frac{\varepsilon}{3}
$$

for any $f \in F$ and $i=1,2, \ldots, l$. In the same way as in the proof of [36, Lemma 4.1 , we see that there exist mutually orthogonal projections $\bar{p}_{1}, \bar{p}_{2}, \ldots, \bar{p}_{l}$ in $F(\mathcal{W})^{* *}$ such that $\bar{p}_{i}$ commutes with $\varphi(C(\Omega))$ and $p_{i} \leq \bar{p}_{i}$. In a similar way as above, there exist a projection $q_{i}$ in $\overline{\psi\left(C_{0}\left(W_{i}\right)\right) F(\mathcal{W}) \psi\left(C_{0}\left(W_{i}\right)\right)}$ such that

$$
\tau_{\omega}\left(p_{i}\right)<\tau_{\omega}\left(q_{i}\right)<\mu\left(W_{i}\right)
$$

and

$$
\left\|q_{i} \psi(f)-f\left(z_{i}\right) q_{i}\right\|<\frac{\varepsilon}{3}, \quad\left\|\psi(f) q_{i}-f\left(z_{i}\right) q_{i}\right\|<\frac{\varepsilon}{3}
$$

for any $f \in F$ and $i=1,2 \ldots, l$. Also, there exist mutually orthogonal projections $\bar{q}_{1}, \bar{q}_{2}, \ldots, \bar{q}_{l}$ in $F(\mathcal{W})^{* *}$ such that $\bar{q}_{i}$ commutes with $\psi(C(\Omega))$ and $q_{i} \leq \bar{q}_{i}$.

For any $i=1,2, \ldots, l$, there exists a subprojection $q_{i}^{\prime}$ of $q_{i}$ such that $q_{i}^{\prime}$ is Murrayvon Neumann equivalent to $p_{i}$ by Proposition 4.1. It follows from Proposition 4.8 that there exists a unitary element $u$ in $F(\mathcal{W})$ such that $u p_{i} u^{*}=q_{i}^{\prime}$ for any $i=1,2, \ldots, l$. Put $p:=1-\sum_{i=1}^{l} p_{i}$ and $q:=1-\sum_{i=1}^{l} q_{i}^{\prime}$. Then we have

$$
0<\tau_{\omega}(p)<\varepsilon \text {. }
$$

Since $(1-p) \varphi(f)=\sum_{i=1}^{l} p_{i} \varphi(f)=\sum_{i=1}^{l} p_{i} \bar{p}_{i} \varphi(f)=\sum_{i=1}^{l} p_{i} \varphi(f) \bar{p}_{i}$, we have

$$
\left\|(1-p) \varphi(f)-\sum_{i=1}^{l} f\left(z_{i}\right) p_{i}\right\|<\frac{\varepsilon}{3}
$$

for any $f \in F$. Moreover, we have

$$
\|[p, \varphi(f)]\|<\frac{2 \varepsilon}{3}
$$

for any $f \in F$. In a similar way, we have

$$
\left\|(1-q) \psi(f)-\sum_{i=1}^{l} f\left(z_{i}\right) q_{i}^{\prime}\right\|<\frac{\varepsilon}{3}, \quad\|[q, \psi(f)]\|<\frac{2 \varepsilon}{3}
$$

for any $f \in F$.

Define unital c.p. maps $\varphi^{\prime}$ and $\psi^{\prime}$ from $C(\Omega)$ to $p F(\mathcal{W}) p$ by

$$
\varphi^{\prime}(f):=p \varphi(f) p, \quad \psi^{\prime}(f):=p u^{*} \psi(f) u p,
$$

and define a unital homomorphism $\sigma$ from $C(\Omega)$ to $(1-p) F(\mathcal{W})(1-p)$ by

$$
\sigma(f):=\sum_{i=1}^{l} f\left(z_{i}\right) p_{i}
$$


Then it is easy to see that $\varphi^{\prime}$ and $\psi^{\prime}$ are $(F, 2 \varepsilon / 3)$-multiplicative maps. We have

$$
\begin{aligned}
\left\|\varphi(f)-\left(\varphi^{\prime}(f)+\sigma(f)\right)\right\| & \leq\|p \varphi(f)-p \varphi(f) p\|+\left\|(1-p) \varphi(f)-\sum_{i=1}^{l} f\left(z_{i}\right) p_{i}\right\| \\
& <\frac{2 \varepsilon}{3}+\frac{\varepsilon}{3}=\varepsilon
\end{aligned}
$$

for any $f \in F$. Also, we have

$$
\left\|\psi(f)-u\left(\psi^{\prime}(f)+\sigma(f)\right) u^{*}\right\|=\left\|\psi(f)-q \psi(f) q-\sum_{i=1}^{l} f\left(z_{i}\right) q_{i}^{\prime}\right\|<\varepsilon
$$

for any $f \in F$. Therefore the proof is complete.

The following theorem is related to [36, Theorem 4.5].

Theorem 5.2. Let $\Omega$ be a compact metrizable space, and let $F_{1}$ be a finite subset of $C(\Omega)$ and $F_{2}$ a finite subset of $\mathcal{W}$, and let $\varepsilon>0$. Then there exist mutually orthogonal positive elements $h_{1}, h_{2}, \ldots, h_{l}$ in $C(\Omega)$ of norm one such that the following holds. For any $\nu>0$, there exist finite subsets $G_{1} \subset C(\Omega), G_{2} \subset \mathcal{W}$ and $\delta>0$ such that the following holds. If $\varphi$ and $\psi$ are unital c.p. maps from $C(\Omega)$ to $M(\mathcal{W})$ such that

$$
\begin{gathered}
\tau\left(\varphi\left(h_{i}\right)\right) \geq \nu, \quad 1 \leq \forall i \leq l, \\
\|[\varphi(f), a]\|<\delta, \quad\|[\psi(f), a]\|<\delta, \quad \forall f \in G_{1}, a \in G_{2}, \\
\|(\varphi(f g)-\varphi(f) \varphi(g)) a\|<\delta, \quad\|(\psi(f g)-\psi(f) \psi(g)) a\|<\delta, \quad \forall f, g \in G_{1}, a \in G_{2}, \\
|\tau(\varphi(f))-\tau(\psi(f))|<\delta, \quad \forall f \in G_{1},
\end{gathered}
$$

then there exists a unitary element $u$ in $\mathcal{W}^{\sim}$ such that

$$
\left\|u \varphi(f) a u^{*}-\psi(f) a\right\|<\varepsilon
$$

for any $f \in F_{1}$ and $a \in F_{2}$.

Proof. We may assume that every element in $F_{2}$ is of norm one. Let $\left\{y_{1}, y_{2}, \ldots, y_{l}\right\}$ be a finite subset of $\Omega$ such that for any $x \in \Omega$, there exists $y_{i} \in\left\{y_{1}, y_{2}, \ldots, y_{l}\right\}$ such that $\left|f(x)-f\left(y_{i}\right)\right|<\varepsilon / 7$ for any $f \in F_{1}$. Choose pairwise disjoint open neighborhoods $W_{1}, W_{2}, \ldots, W_{l}$ of $y_{1}, y_{2}, \ldots, y_{l}$ respectively such that if $x \in W_{i}$ and $f \in F_{1}$, then $\left|f(x)-f\left(y_{i}\right)\right|<\varepsilon / 7$. For any $i=1,2, \ldots, l$, take a positive element $h_{i} \in C_{0}\left(W_{i}\right)$ of norm one. We shall show that $h_{1}, h_{2}, \ldots, h_{l}$ have the desired property. On the contrary, suppose that $h_{1}, h_{2}, \ldots, h_{l}$ did not have the desired property. Then there exists a positive number $\nu$ satisfying the following: For any $n \in \mathbb{N}$, there exist unital c.p. maps $\varphi_{n}, \psi_{n}: C(\Omega) \rightarrow M(\mathcal{W})$ such that

$$
\begin{array}{rlrl}
\tau\left(\varphi_{n}\left(h_{i}\right)\right) & \geq \nu, & & 1 \leq \forall i \leq l, \\
\left\|\left[\varphi_{n}(f), a\right]\right\| \rightarrow 0, \quad\left\|\left[\psi_{n}(f), a\right]\right\| & \rightarrow 0, & \left\|\left(\varphi_{n}(f g)-\varphi_{n}(f) \varphi_{n}(g)\right) a\right\| \rightarrow 0, \\
\left\|\left(\psi_{n}(f g)-\psi_{n}(f) \psi_{n}(g)\right) a\right\| & \rightarrow 0, & \left|\tau\left(\varphi_{n}(f)\right)-\tau\left(\psi_{n}(f)\right)\right| \rightarrow 0
\end{array}
$$

as $n \rightarrow \infty$ for any $f, g \in C(\Omega), a \in \mathcal{W}$ and

$$
\max _{f \in F_{1}, a \in F_{2}}\left\|u \varphi_{n}(f) a u^{*}-\psi_{n}(f) a\right\| \geq \varepsilon
$$

for any unitary element $u$ in $\mathcal{W}^{\sim}$.

Define homomorphisms $\varphi$ and $\psi$ from $C(\Omega)$ to $F(\mathcal{W})$ by

$$
\varphi(f):=\left[\left(\varphi_{n}(f)\right)_{n}\right], \quad \psi(f):=\left[\left(\psi_{n}(f)\right)_{n}\right]
$$

for any $f \in C(\Omega)$, and define homomorphisms $\Phi$ and $\Psi$ from $C(\Omega) \otimes \mathcal{W}$ to $\mathcal{W}^{\omega}$ by

$$
\Phi:=\rho \circ\left(\varphi \otimes \operatorname{id}_{\mathcal{W}}\right), \quad \Psi:=\rho \circ\left(\psi \otimes \operatorname{id}_{\mathcal{W}}\right) .
$$

Note that we have

$$
\tau_{\omega}\left(\varphi\left(h_{i}\right)\right) \geq \nu
$$


for any $i=1,2, \ldots, l$ and

$$
\tau_{\omega} \circ \varphi=\tau_{\omega} \circ \psi
$$

Applying Corollary 3.8 to $F_{1}, F_{2}$ and $\varepsilon / 7$, we obtain finite subsets $G_{1} \subset C(\Omega)$ $G_{2} \subset \mathcal{W}, m \in \mathbb{N}$ and $\delta>0$. Put

$$
F_{1}^{\prime}:=F_{1} \cup G_{1} \cup\left\{h_{1}, h_{2}, \ldots, h_{l}\right\}, \quad \varepsilon^{\prime}:=\min \left\{\varepsilon / 7, \delta, \nu /\left(m^{2}+2\right)\right\} .
$$

Applying Lemma 5.1 to $F_{1}^{\prime}, \varepsilon^{\prime}, \varphi$ and $\psi$, there exist a projection $p \in F(\mathcal{W})$, $\left(F^{\prime}, \varepsilon^{\prime}\right)$-multiplicative unital c.p. maps $\varphi^{\prime}$ and $\psi^{\prime}$ from $C(\Omega)$ to $p F(\mathcal{W}) p$ and a unital homomorphism $\sigma$ from $C(\Omega)$ to $(1-p) F(\mathcal{W})(1-p)$ with finite-dimensional range such that

$$
0<\tau_{\omega}(p)<\varepsilon^{\prime}, \quad \varphi \sim_{F_{1}^{\prime}, \varepsilon^{\prime}} \varphi^{\prime} \oplus \sigma, \quad \psi \sim_{F_{1}^{\prime}, \varepsilon^{\prime}} \psi^{\prime} \oplus \sigma .
$$

Define c.p. maps $\Phi^{\prime}$ and $\Psi^{\prime}$ from $C(\Omega) \otimes \mathcal{W}$ to $\mathcal{W}_{p}^{\omega}$ by

$$
\Phi^{\prime}:=\rho \circ\left(\varphi^{\prime} \otimes \operatorname{id}_{\mathcal{W}}\right), \quad \Psi^{\prime}:=\rho \circ\left(\psi^{\prime} \otimes \operatorname{id}_{\mathcal{W}}\right)
$$

and define a homomorphism $\Sigma$ from $C(\Omega) \otimes \mathcal{W}$ to $\mathcal{W}_{1-p}^{\omega}$ by

$$
\Sigma:=\rho \circ\left(\sigma \otimes \operatorname{id}_{\mathcal{W}}\right) .
$$

Since every unitary element in $F(\mathcal{W})$ can be lifted to a unitary element in $\mathcal{W}_{\omega}^{\sim}$ by Corollary 4.10

$$
\Phi \sim_{F_{1} \odot F_{2}, \frac{\varepsilon}{7}} \Phi^{\prime} \oplus \Sigma, \quad \Psi \sim_{F_{1} \odot F_{2}, \frac{\varepsilon}{7}} \Psi^{\prime} \oplus \Sigma .
$$

It can be easily checked that $\Phi^{\prime}$ and $\Psi^{\prime}$ are contractive $\left(G_{1} \odot G_{2}, \delta\right)$-multiplicative maps. By Corollary 3.8 , there exist a unitary element $U$ in $M_{m^{2}+1}\left(\mathcal{W}_{p}^{\omega}\right)^{\sim}$ and $z_{1}, z_{2}, \ldots, z_{m} \in \Omega$ such that

$$
\begin{aligned}
\| U^{*}(\Phi^{\prime}(f \otimes a) \oplus \overbrace{\left.\bigoplus_{k=1}^{m} f\left(z_{k}\right) \rho(p \otimes a) \oplus \cdots \oplus \bigoplus_{k=1}^{m} f\left(z_{k}\right) \rho(p \otimes a)\right)} & \overbrace{\bigoplus_{\bigoplus_{k=1}^{m} f\left(z_{k}\right) \rho(p \otimes a) \oplus \cdots \oplus \bigoplus_{k=1}^{m} f\left(z_{k}\right) \rho(p \otimes a)}^{m} \|}^{m}<\frac{\varepsilon}{7} \\
& -\Psi^{\prime}(f \otimes a)
\end{aligned}
$$

for any $f \in F_{1}$ and $a \in F_{2}$.

For any homomorphism $\gamma: C(\Omega) \rightarrow F(\mathcal{W})$, let $\mu_{\gamma}$ denote the probability measure on $\Omega$ corresponding to $\tau_{\omega} \circ \gamma$. For any $i=1,2, \ldots, l$, we have

$\mu_{\sigma}\left(W_{i}\right) \geq \tau_{\omega}\left(\sigma\left(h_{i}\right)\right)>\tau_{\omega}\left(\varphi\left(h_{i}\right)\right)-\tau_{\omega}\left(\varphi^{\prime}\left(h_{i}\right)\right)-\varepsilon^{\prime} \geq \nu-\tau_{\omega}(p)-\varepsilon^{\prime}>\nu-2 \varepsilon^{\prime} \geq m^{2} \varepsilon^{\prime}$.

Hence we see that there exists a homomorphism $\sigma^{\prime}: C(\Omega) \rightarrow(1-p) F(\mathcal{W})(1-p)$ with finite-dimensional range such that

$$
\left\|\sigma(f)-\sigma^{\prime}(f)\right\|<\frac{\varepsilon}{7}
$$

for any $f \in F_{1}$ and $\mu_{\sigma^{\prime}}\left(\left\{y_{i}\right\}\right)>m^{2} \varepsilon^{\prime}$ for any $i=1,2, \ldots, l$ because of the property of $W_{i}$. Using Proposition 4.1 we see that there exist mutually orthogonal projections $\left\{p_{j, k}\right\}_{j, k=1}^{m}$ in $(1-p) F(\mathcal{W})(1-p)$ and a homomorphism $\sigma^{\prime \prime}: C(\Omega) \rightarrow(1-p-$ q) $F(\mathcal{W})(1-p-q)$ where $q=\sum_{j, k=1}^{m} p_{j, k}$ such that

$$
\left\|\sigma^{\prime}(f)-\left(\sum_{j=1}^{m} \sum_{k=1}^{m} f\left(z_{k}\right) p_{j, k}+\sigma^{\prime \prime}(f)\right)\right\|<\frac{\varepsilon}{7}
$$

for any $f \in F_{1}$ and $p_{j, k}$ is Murray-von Neumann equivalent to $p$ for any $j, k=$ $1,2, \ldots, m$ because of the property of $\left\{y_{1}, y_{2}, \ldots, y_{l}\right\}$. 
Since $\Phi^{\prime}(f \otimes a)+\sum_{j=1}^{m} \sum_{k=1}^{m} f\left(z_{k}\right) \rho\left(p_{j, k} \otimes a\right)$ in $\mathcal{W}_{p+q}^{\omega}$ can be regarded as an element in $M_{m^{2}+1}\left(\mathcal{W}_{p}^{\omega}\right)^{\sim}$, there exists a unitary element $\widehat{U}$ in $\left(\mathcal{W}_{p+q}^{\omega}\right)^{\sim}$ such that

$$
\begin{aligned}
\| \widehat{U}^{*}\left(\Phi^{\prime}(f \otimes a)+\sum_{j=1}^{m} \sum_{k=1}^{m}\right. & \left.f\left(z_{k}\right) \rho\left(p_{j, k} \otimes a\right)\right) \widehat{U} \\
& \quad-\left(\Psi^{\prime}(f \otimes a)+\sum_{j=1}^{m} \sum_{k=1}^{m} f\left(z_{k}\right) \rho\left(p_{j, k} \otimes a\right)\right) \|<\frac{\varepsilon}{7}
\end{aligned}
$$

by the argument above. Note that we may assume that $\widehat{U}=\left(a_{n}\right)_{n}+1$ for some $\left(a_{n}\right)_{n} \in \mathcal{W}_{p+q}^{\omega}$. Let $V$ be a trivial unitary extension in $\left(\mathcal{W}^{\omega}\right)^{\sim}$ from $\widehat{U}$. Then we have

$$
\begin{aligned}
\| V^{*}\left(\Phi^{\prime}(f \otimes a)+\right. & \left.\sum_{j=1}^{m} \sum_{k=1}^{m} f\left(z_{k}\right) \rho\left(p_{j, k} \otimes a\right)+\sigma^{\prime \prime}(f)\right) V \\
& -\left(\Psi^{\prime}(f \otimes a)+\sum_{j=1}^{m} \sum_{k=1}^{m} f\left(z_{k}\right) \rho\left(p_{j, k} \otimes a\right)+\sigma^{\prime \prime}(f)\right) \|<\frac{\varepsilon}{7} .
\end{aligned}
$$

Let $\Sigma^{\prime}$ and $\Sigma^{\prime \prime}$ be homomorphisms from $C(\Omega) \otimes \mathcal{W}$ to $\mathcal{W}_{1-p}^{\omega}$ such that

$$
\Sigma^{\prime}(f \otimes a)=\rho\left(\sigma^{\prime}(f) \otimes a\right), \quad \Sigma^{\prime \prime}(f \otimes a)=\rho\left(\sum_{j=1}^{m} \sum_{k=1}^{m}\left(f\left(z_{k}\right) p_{j, k}+\sigma^{\prime \prime}(f)\right) \otimes a\right)
$$

for any $f \in C(\Omega)$ and $a \in \mathcal{W}$. Then we have

$$
\begin{aligned}
\Phi & \sim_{F_{1} \odot F_{2}, \frac{\varepsilon}{7}} \Phi^{\prime} \oplus \Sigma \sim_{F_{1} \odot F_{2}, \frac{\varepsilon}{7}} \Phi^{\prime} \oplus \Sigma^{\prime} \sim_{F_{1} \odot F_{2}, \frac{\varepsilon}{7}} \Phi^{\prime} \oplus \Sigma^{\prime \prime} \sim_{F_{1} \odot F_{2}, \frac{\varepsilon}{7}} \Psi^{\prime} \oplus \Sigma^{\prime \prime} \\
& \sim_{F_{1} \odot F_{2}, \frac{\varepsilon}{7}} \Psi^{\prime} \oplus \Sigma^{\prime} \sim_{F_{1} \odot F_{2}, \frac{\varepsilon}{7}} \Psi^{\prime} \oplus \Sigma \sim_{F_{1} \odot F_{2}, \frac{\varepsilon}{7}} \Psi .
\end{aligned}
$$

Therefore there exists a unitary element $\left(w_{n}\right)_{n}$ in $\left(\mathcal{W}^{\omega}\right)^{\sim}$ such that

$$
\left\|\left(w_{n}\right)_{n} \Phi(f \otimes a)\left(w_{n}\right)_{n}^{*}-\Psi(f \otimes a)\right\|<\varepsilon
$$

for any $f \in F_{1}$ and $a \in F_{2}$. Note that we may assume that $w_{n}$ is a unitary element in $\mathcal{W}^{\sim}$ for any $n \in \mathbb{N}$. Taking a sufficiently large $n$, we obtain a contradiction. Consequently, the proof is complete.

Let $\mathbb{T}$ be the unit circle in the complex plane. We denote by $\iota$ the identity function on $\mathbb{T}$, that is, $\iota(z)=z$ for any $z \in \mathbb{T}$.

Theorem 5.3. Let $u$ and $v$ be unitaries in $F(\mathcal{W})$ such that $\tau_{\omega}(f(u))>0$ for any $f \in C(\mathbb{T})_{+} \backslash\{0\}$. Then there exists a unitary element $w$ in $F(\mathcal{W})$ such that $w u w^{*}=v$ if and only if $\tau_{\omega}(f(u))=\tau_{\omega}(f(v))$ for any $f \in C(\mathbb{T})$.

Proof. The only if part is obvious. We will show the if part. By Corollary 4.10, there exist unitaries $\left(u_{n}\right)_{n}$ and $\left(v_{n}\right)_{n}$ in $\mathcal{W}_{\omega}^{\sim}$ such that $u=\left[\left(u_{n}\right)_{n}\right]$ and $v=\left[\left(v_{n}\right)_{n}\right]$. For any $n \in \mathbb{N}$, define unital homomorphisms $\varphi_{n}$ and $\psi_{n}$ from $C(\mathbb{T})$ to $\mathcal{W}^{\sim}$ by $\varphi_{n}(f):=f\left(u_{n}\right)$ and $\psi_{n}(f):=f\left(v_{n}\right)$, respectively. Then we have

$$
\begin{gathered}
\left|\tau\left(\varphi_{n}(f)\right)-\tau_{\omega}(f(u))\right| \rightarrow 0, \quad\left\|\left[\varphi_{n}(f), a\right]\right\| \rightarrow 0, \quad\left\|\left[\psi_{n}(f), a\right]\right\| \rightarrow 0, \\
\left|\tau\left(\varphi_{n}(f)\right)-\tau\left(\psi_{n}(f)\right)\right| \rightarrow 0
\end{gathered}
$$

as $n \rightarrow \omega$ for any $f \in C(\mathbb{T})$ and $a \in \mathcal{W}$.

Let $F_{1}:=\{1, \iota\} \subset C(\mathbb{T})$, and let $\left\{F_{2, k}\right\}_{k \in \mathbb{N}}$ be a sequence of finite subsets of $\mathcal{W}$ such that $F_{2, k} \subset F_{2, k+1}$ and $\mathcal{W}=\overline{\bigcup_{k \in \mathbb{N}} F_{2, k}}$. For any $k \in \mathbb{N}$, applying Theorem 5.2 to $F_{1}, F_{2, k}$ and $1 / k$, we obtain mutually orthogonal positive elements $h_{1, k}, h_{2, k}, \ldots, h_{l(k), k}$ in $C(\mathbb{T})$ of norm one. Let

$$
\nu_{k}:=\frac{1}{2} \min \left\{\tau_{\omega}\left(h_{1, k}(u)\right), \tau_{\omega}\left(h_{2, k}(u)\right), \ldots, \tau_{\omega}\left(h_{l(k), k}(u)\right)\right\}>0 .
$$


Applying Theorem 5.2 to $\nu_{k}$, we obtain finite subsets $G_{1, k} \subset C(\mathbb{T}), G_{2, k} \subset \mathcal{W}$ and $\delta_{k}>0$. We may assume that $G_{1, k} \subset G_{1, k+1}, G_{2, k} \subset G_{2, k+1}$ and $\delta_{k}>\delta_{k+1}$. It can be easily checked that there exists a sequence $\left\{X_{k}\right\}_{k \in \mathbb{N}}$ of elements in $\omega$ such that $X_{k} \supset X_{k+1}$ and for any $n \in X_{k}$,

$$
\begin{gathered}
\left|\tau\left(\varphi_{n}\left(h_{i, k}\right)\right)-\tau_{\omega}\left(h_{i, k}(u)\right)\right|<\frac{\tau_{\omega}\left(h_{i, k}(u)\right)}{2}, \quad 1 \leq \forall i \leq l(k), \\
\left\|\left[\varphi_{n}(f), a\right]\right\|<\delta_{k}, \quad\left\|\left[\psi_{n}(f), a\right]\right\|<\delta_{k}, \quad \forall f \in G_{1, k}, a \in G_{2, k}, \\
\left|\tau\left(\varphi_{n}(f)\right)-\tau\left(\psi_{n}(f)\right)\right|<\delta_{k}, \quad \forall f \in G_{1, k} .
\end{gathered}
$$

Since we have $\tau\left(\varphi_{n}\left(h_{i, k}\right)\right) \geq \nu_{k}$ by the above, Theorem 5.2 implies that for any $n \in X_{k}$, there exists a unitary element $w_{k, n}$ in $\mathcal{W}^{\sim}$ such that

$$
\left\|w_{k, n} \varphi_{n}(f) a w_{k, n}^{*}-\psi_{n}(f) a\right\|<\frac{1}{k}
$$

for any $f \in F_{1}$ and $a \in F_{2, k}$. Since $F_{1}=\{1, \iota\}$, we have

for any $n \in X_{k}$ and $a \in F_{2, k}$. Let

$$
\left\|\left[w_{k, n}, a\right]\right\|<\frac{1}{k}, \quad\left\|w_{k, n} u_{n} a w_{k, n}^{*}-v_{n} a\right\|<\frac{1}{k}
$$

$$
w_{n}:=\left\{\begin{array}{cl}
1 & \text { if } n \notin X_{1} \\
w_{k, n} & \text { if } n \in X_{k} \backslash X_{k+1} \quad(k \in \mathbb{N})
\end{array} .\right.
$$

Then we have

$$
\left\|\left[w_{n}, a\right]\right\| \rightarrow 0, \quad\left\|w_{n} u_{n} w_{n}^{*} a-v_{n} a\right\| \rightarrow 0
$$

as $n \rightarrow \omega$ for any $a \in \mathcal{W}$. Therefore $\left[\left(w_{n}\right)_{n}\right]$ is a unitary element in $F(\mathcal{W})$ and

$$
\left[\left(w_{n}\right)_{n}\right] u\left[\left(w_{n}\right)_{n}\right]^{*}=v \text {. }
$$

Hiroki Matui told us the following lemma.

Lemma 5.4. For any faithful tracial state $\tau_{0}$ on $C(\mathbb{T})$, there exists a unital homomorphism $\varphi$ from $C(\mathbb{T})$ to $M_{2}$ such that $\tau_{0}=\tau^{\prime} \circ \varphi$ where $\tau^{\prime}$ is the unique tracial state on $M_{2 \infty}$.

Proof. We identify $C(\mathbb{T})$ with $\{f \in C([0,1]) \mid f(0)=f(1)\}$. Note that $\tau_{0}$ extends to a faithful tracial state $\tilde{\tau}_{0}$ on $C([0,1])$. By [50, Theorem 2.1 (i)], there exists a unital homomorphism $\psi$ from $C([0,1])$ to $\mathcal{Z}$ such that $\tilde{\tau}_{0}=\tau_{\mathcal{Z}} \circ \psi$, where $\tau_{\mathcal{Z}}$ is the unique tracial state on $\mathcal{Z}$. Define a unital homomorphism $\varphi$ from $C(\mathbb{T})$ to $M_{2} \otimes \mathcal{Z}$ by $\varphi:=\left.1 \otimes \psi\right|_{C(\mathbb{T})}$. Since $M_{2}$ is $\mathcal{Z}$-stable, we obtain the conclusion.

Note that we identify $F(\mathcal{W})$ with $F\left(\mathcal{W} \otimes M_{2 \infty}\right)$ in the following lemmas.

Lemma 5.5. Let $u$ be a unitary element in $F(\mathcal{W})$ such that $\tau_{\omega}(f(u))>0$ for any $f \in C(\mathbb{T})_{+} \backslash\{0\}$. Then there exist a unitary element $\left(v_{n}\right)_{n}$ in $\left(M_{2} \infty\right)_{\omega}$ and a unitary element $w$ in $F(\mathcal{W})$ such that

$$
w u w^{*}=\left[\left(h_{n} \otimes v_{n}\right)_{n}\right]
$$

where $\left\{h_{n}\right\}_{n \in \mathbb{N}}$ is an approximate unit for $\mathcal{W}$.

Proof. By Lemma 5.4, there exists a unital homomorphism $\varphi$ from $C(\mathbb{T})$ to $M_{2} \infty$ such that $\tau^{\prime}(\varphi(f))=\tau_{\omega}(f(u))$ for any $f \in C(\mathbb{T})$, where $\tau^{\prime}$ is the unique tracial state on $M_{2^{\infty}}$. For any $n \in \mathbb{N}$, let

$$
v_{n}:=\overbrace{1 \otimes \cdots \otimes 1}^{n} \otimes \varphi(\iota) \otimes 1 \otimes \cdots \in \bigotimes_{n \in \mathbb{N}} M_{2^{\infty}} \cong M_{2^{\infty}} .
$$


Then $\left(v_{n}\right)_{n}$ is a unitary element in $\left(M_{2} \infty\right)_{\omega}$ and we have

$$
\tau_{\omega}(f(u))=\tau_{\omega}\left(f\left(\left[\left(h_{n} \otimes v_{n}\right)_{n}\right]\right)\right)
$$

for any $f \in C(\mathbb{T})$. Therefore we obtain the conclusion by Theorem 5.3 .

For a Lipschitz continuous map $U$, we denote by $\operatorname{Lip}(U)$ its Lipschitz constant.

Lemma 5.6. Let $u$ be a unitary element in $F(\mathcal{W})$ such that $\tau_{\omega}(f(u))>0$ for any $f \in C(\mathbb{T})_{+} \backslash\{0\}$, and let $z_{0} \in \mathbb{T}$. Then there exists a continuous path of unitaries $U:[0,1] \rightarrow F(\mathcal{W})$ such that

$$
U(0)=z_{0} 1, \quad U(1)=u, \quad \operatorname{Lip}(U) \leq \pi .
$$

Proof. Let $\left\{h_{n}\right\}_{n \in \mathbb{N}}$ be an approximate unit for $A$. By Lemma 5.5. there exist a unitary element $\left(v_{n}\right)_{n}$ in $\left(M_{2 \infty}\right)_{\omega}$ and a unitary element $w$ in $F(\mathcal{W})$ such that $w u w^{*}=\left[\left(h_{n} \otimes v_{n}\right)_{n}\right]$. There exists a continuous path of unitaries $V:[0,1] \rightarrow$ $\left(M_{2} \infty\right)_{\omega}$ such that

$$
V(0)=z_{0} 1, \quad V(1)=\left(v_{n}\right)_{n}, \quad \operatorname{Lip}(U) \leq \pi .
$$

(See, for example, [17, Lemma 1].) For any $t \in[0,1]$, let $\left(v_{n}(t)\right)_{n}$ be a representative of $V(t)$. Define a continuous path of unitaries $U:[0,1] \rightarrow F(\mathcal{W})$ by

$$
U(t):=w^{*}\left[\left(h_{n} \otimes v_{n}(t)\right)_{n}\right] w
$$

for any $t \in[0,1]$. Then $U$ has the desired property.

The following theorem is the main theorem in this section.

Theorem 5.7. Let $u$ be a unitary element in $F(\mathcal{W})$. There exists a continuous path of unitaries $U:[0,1] \rightarrow F(\mathcal{W})$ such that

$$
U(0)=1, \quad U(1)=u, \quad \operatorname{Lip}(U) \leq 2 \pi .
$$

Proof. Let $\varepsilon>0$ and $\delta>0$. We denote by $\mu$ the probability measure on $\mathbb{T}$ corresponding to $f \mapsto \tau_{\omega}(f(u))$. Since $\mu(\mathbb{T})=1$, there exists an element $z_{0}$ in $\mathbb{T}$ such that $\mu\left(\left\{z \in \mathbb{T}|| z-z_{0} \mid<\delta\right\}\right)>0$. Let $h$ be a positive element in $C(\mathbb{T})$ such that

$$
\left\{z \in \mathbb{T}|| z-z_{0} \mid<\delta\right\} \subset \overline{\operatorname{supp} h} \subset\left\{z \in \mathbb{T}|| z-z_{0} \mid<2 \delta\right\} .
$$

Then we have $d_{\tau_{\omega}}(h(u))>0$. Proposition 4.2 implies that there exists projection $p$ in $\overline{h(u) F(\mathcal{W}) h(u)}$ such that $\tau_{\omega}(p)>0$. Similar arguments as in the proof of 6 , Lemma 1.7] show that there exist a unitary element $u^{\prime}$ in $(1-p) F(\mathcal{W})(1-p)$ and a continuous path of unitaries $V_{1}:[0,1] \rightarrow F(\mathcal{W})$ such that

$$
V_{1}(0)=u^{\prime}+z_{0} p, \quad V_{1}(1)=u, \quad \operatorname{Lip}\left(V_{1}\right)<\varepsilon .
$$

Indeed, we have

$$
\left\|u-\left((1-p) u(1-p)+z_{0} p\right)\right\|<6 \delta
$$

and, taking a sufficiently small $\delta>0$ and using polar decomposition, we obtain a continuous path of unitaries $V_{1}$ as above. By Lemma 5.4 and the proof of Lemma 5.5. it is easy to see that there exist a unitary element $v$ in $F(\mathcal{W})$ such that $\tau_{\omega}(f(v))>0$ for any $f \in C(\mathbb{T})_{+} \backslash\{0\}$. Using Lemma 5.6. Lemma 3.6 and the slow reindexation trick, we may assume that $v p=p v$ and $\tau_{\omega}(p v)=\tau_{\omega}(p) \tau_{\omega}(v)$, and we see that there exists a continuous path of unitaries $V_{2}:[0,1] \rightarrow F(\mathcal{W})$ such that

$$
V_{2}(0)=u^{\prime}+v p, \quad V_{2}(1)=u^{\prime}+z_{0} p, \quad \operatorname{Lip}\left(V_{2}\right) \leq \pi .
$$

(See, for example, 44 for the slow reindexation trick.) Since we have $\tau_{\omega}\left(f\left(u^{\prime}+\right.\right.$ $v p))=\tau_{\omega}\left(f\left(u^{\prime}\right)\right)+\tau_{\omega}(f(v)) \tau_{\omega}(p)>0$ for any $f \in C(\mathbb{T})_{+} \backslash\{0\}$, it follows from Lemma 5.6 that there exists a continuous path of unitaries $V_{3}:[0,1] \rightarrow F(\mathcal{W})$ such that

$$
V_{3}(0)=1, \quad V_{3}(1)=u^{\prime}+v p, \quad \operatorname{Lip}\left(V_{3}\right) \leq \pi
$$


Connecting $V_{1}, V_{2}$ and $V_{3}$, we obtain a continuous path of unitaries $U:[0,1] \rightarrow$ $F(\mathcal{W})$ such that

$$
U(0)=1, \quad U(1)=u, \quad \operatorname{Lip}(U)<2 \pi+\varepsilon .
$$

We obtain the conclusion by the usual diagonal argument.

We shall show another application of Theorem 5.2

Theorem 5.8. Let $p$ and $q$ be projections in $F(\mathcal{W})$ such that $0<\tau_{\omega}(p)<1$. Then $p$ and $q$ are unitarily equivalent if and only if $\tau_{\omega}(p)=\tau_{\omega}(q)$.

Proof. The only if part is obvious. We will show the if part. Note that the $\mathrm{C}^{*}$ algebra generated by 1 and $p$ is isomorphic to $C(\{0,1\}) \cong \mathbb{C}^{2}$. Since $\tau_{\omega}(p)>0$ and $\tau_{\omega}(1-p)>0$, we have $\tau_{\omega}(f(p))>0$ for any $f \in C(\{0,1\})_{+} \backslash\{0\}$. Also, we have $\tau_{\omega}(f(p))=\tau_{\omega}(f(q))$ for any $f \in C(\{0,1\})$. There exist positive contractions $\left(p_{n}\right)_{n}$ and $\left(q_{n}\right)_{n}$ in $\mathcal{W}_{\omega}$ such that $p=\left[\left(p_{n}\right)_{n}\right]$ and $q=\left[\left(q_{n}\right)_{n}\right]$. For any $n \in \mathbb{N}$, define unital c.p. maps $\varphi_{n}$ and $\psi_{n}$ from $C(\{0,1\}) \cong \mathbb{C}^{2}$ to $\mathcal{W}^{\sim}$ by $\varphi_{n}\left(\left(\lambda_{1}, \lambda_{2}\right)\right):=$ $\lambda_{1} p_{n}+\lambda_{2}\left(1-p_{n}\right)$ and $\psi_{n}\left(\left(\lambda_{1}, \lambda_{2}\right)\right):=\lambda_{1} q_{n}+\lambda_{2}\left(1-q_{n}\right)$, respectively. Then we have

$$
\begin{gathered}
\left|\tau\left(\varphi_{n}(f)\right)-\tau_{\omega}(f(p))\right| \rightarrow 0, \quad\left\|\left[\varphi_{n}(f), a\right]\right\| \rightarrow 0, \quad\left\|\left[\psi_{n}(f), a\right]\right\| \rightarrow 0, \\
\left\|\left(\varphi_{n}(f g)-\varphi_{n}(f) \varphi_{n}(g)\right) a\right\| \rightarrow 0, \quad\left\|\left(\psi_{n}(f g)-\psi_{n}(f) \psi_{n}(g)\right) a\right\| \rightarrow 0, \\
\left|\tau\left(\varphi_{n}(f)\right)-\tau\left(\psi_{n}(f)\right)\right| \rightarrow 0
\end{gathered}
$$

as $n \rightarrow \omega$ for any $f, g \in C(\{0,1\}), a \in \mathcal{W}$. Therefore the rest of proof is same as the proof of Theorem 5.3

The following corollary is an answer to [22, Question 2.14].

Corollary 5.9. The unit 1 in $F(\mathcal{W})$ is infinite.

Proof. By Proposition 4.2, there exist mutually orthogonal non-zero projections $p$ and $q$ in $F(\mathcal{W})$ such that $\tau_{\omega}(p)=1 / 2$ and $\tau_{\omega}(q)=0$. Theorem 5.8 implies that $1-p$ is unitarily equivalent to $1-(p+q)$. Therefore $1=(1-p)+p$ is Murray-von Neumann equivalent to $(1-(p+q))+p=1-q$. Hence 1 is Murray-von Neumann equivalent to a proper subprojection. Consequently, 1 is infinite.

The corollary above suggests the following question.

Question 5.10. Let $A$ be a simple separable ( $\mathcal{Z}$-stable) stably projectionless $\mathrm{C}^{*}$ algebra. Is 1 infinite in $F(A)$ ?

Yuhei Suzuki suggested the following corollary.

Corollary 5.11. The tracial state $\tau_{\omega}$ on $F(\mathcal{W})$ induces an order isomorphism $\tau_{\omega *}$ from $K_{0}(F(\mathcal{W}))$ onto $\mathbb{R}$.

Proof. Let $p$ and $q$ be projections in $M_{\infty}(F(\mathcal{W}))$ such that $\tau_{\omega *}([p]-[q])=0$. Using Proposition 4.2 and Proposition 4.1, we see that there exist a natural number $k$, projections $p^{\prime}, q^{\prime}$ and $r$ in $F(\mathcal{W})$ such that $[p]=\left[p^{\prime}\right]+k[1]+[r],[q]=\left[q^{\prime}\right]+k[1]+[r]$ and $\tau_{\omega}\left(p^{\prime}\right)=\tau_{\omega}\left(q^{\prime}\right)<1$. Moreover, we may assume that there exists a projection $e$ in $F(\mathcal{W})$ such that $p^{\prime}, q^{\prime} \in e F(\mathcal{W}) e$ and $\tau_{\omega}\left(p^{\prime}\right)<\tau_{\omega}(e)<1$. Theorem 5.8 implies that $p^{\prime}+1-e$ is unitarily equivalent to $q^{\prime}+1-e$ because we have $0<\tau_{\omega}\left(p^{\prime}+1-e\right)<1$. Then $\left[p^{\prime}\right]=\left[q^{\prime}\right]$, and hence $[p]=[q]$. Therefore $\tau_{\omega *}$ is injective. It follows from Proposition 4.2 that $\tau_{\omega *}$ is surjective. Consequently, $\tau_{\omega *}$ is an order isomorphism from $K_{0}(F(\mathcal{W}))$ onto $\mathbb{R}$. 


\section{Rohlin type theOREM}

In this section we shall show that every trace scaling automorphism of $\mathcal{W} \otimes \mathbb{K}$ has the Rohlin property.

Definition 6.1. Let $A$ be a separable $\mathrm{C}^{*}$-algebra, and let $\alpha$ be an automorphism of $A$. We say that $\alpha$ has the Rohlin property if for any $k \in \mathbb{N}$, there exist projections $\left\{p_{1, i}\right\}_{i=0}^{k-1}$ and $\left\{p_{2, j}\right\}_{j=0}^{k}$ in $F(A)$ such that

$$
\sum_{i=0}^{k-1} p_{1, i}+\sum_{j=0}^{k} p_{2, j}=1, \quad \alpha\left(p_{1, i}\right)=p_{1, i+1}, \quad \alpha\left(p_{2, j}\right)=p_{2, j+1}
$$

for any $i=0,1, \ldots, k-2$ and $j=0,1, \ldots, k-1$.

If $A$ is unital, then the definition above coincides with the usual definition (see, for example, [27]).

We identify $F(\mathcal{W} \otimes \mathbb{K})$ with $F(\mathcal{W})$. We denote by the same symbol $\tau_{\omega}$ the unique tracial state on $F(\mathcal{W} \otimes \mathbb{K})$ for simplicity. Note that for any $\left[\left(x_{n}\right)_{n}\right] \in F(\mathcal{W} \otimes \mathbb{K})$, $\tau_{\omega}\left(\left[\left(x_{n}\right)_{n}\right]\right)=\lim _{n \rightarrow \omega} \tau \otimes \operatorname{Tr}\left(x_{n} h\right) / \tau \otimes \operatorname{Tr}(h)$ for some $h \in \operatorname{Ped}(\mathcal{W} \otimes \mathbb{K})_{+} \backslash\{0\}$ (see Remark 2.4). The following lemma is a variant of [37, Theorem 3.4].

Lemma 6.2. Let $\alpha$ be a trace scaling automorphism of $\mathcal{W} \otimes \mathbb{K}$. Then for any $k \in \mathbb{N}$, there exists a positive contraction $f$ in $F(\mathcal{W} \otimes \mathbb{K})$ such that

$$
\tau_{\omega}(f)=\frac{1}{k}, \quad f \alpha^{j}(f)=0
$$

for any $j=1,2, \ldots, k-1$.

Proof. Note that $\pi_{\tau \otimes \operatorname{Tr}}(\mathcal{W} \otimes \mathbb{K})^{\prime \prime}$ is the AFD factor of type $\mathrm{II}_{\infty}$ and $\tilde{\alpha}$ is a trace scaling automorphism. Hence it follows from [5, Lemma 5] and [5, Theorem 1.2.5] that there exist projections $\left\{\tilde{p}_{j}\right\}_{j=1}^{k}$ in $\left(\pi_{\tau \otimes \operatorname{Tr}}(\mathcal{W} \otimes \mathbb{K})^{\prime \prime}\right)_{\omega}$ such that

$$
\sum_{j=1}^{k} \tilde{p}_{j}=1, \quad \tilde{\alpha}\left(\tilde{p}_{j}\right)=\tilde{p}_{j+1}
$$

for any $j=1,2, \ldots, k-1$. Proposition 2.3 implies that there exists a positive contraction $e$ in $F(\mathcal{W} \otimes \mathbb{K})$ such that $\varrho_{\mathcal{W} \otimes \mathbb{K}}(e)=\tilde{p}_{1}$. It is easy to see that $\tau_{\omega}(e)=$ $1 / k$. Let $\left(e_{n}\right)_{n}$ be a representative of $e$. Then

$$
\left\|\pi_{\tau \otimes \operatorname{Tr}}\left(e_{n} \alpha^{j}\left(e_{n}\right)\right) \hat{h}\right\|_{2} \rightarrow 0
$$

as $n \rightarrow \omega$ for any $h \in \operatorname{Ped}(\mathcal{W} \otimes \mathbb{K})$ and $j=1,2, \ldots, k-1$. By similar arguments as in the proof of [37, Proposition 3.3], one can prove the lemma. Indeed, put

$$
e_{n}^{\prime}:=e_{n}^{\frac{1}{2}}\left(\sum_{j=1}^{k-1} \alpha^{j}\left(e_{n}\right)\right) e_{n}^{\frac{1}{2}}
$$

Then $\left\|\pi_{\tau \otimes \operatorname{Tr}}\left(e_{n}^{\prime}\right) \hat{h}\right\|_{2} \rightarrow 0$ as $n \rightarrow \omega$ for any $h \in \operatorname{Ped}(\mathcal{W} \otimes \mathbb{K})$. For any $\varepsilon>0$, define

$$
g_{\varepsilon}(t):=\left\{\begin{array}{cl}
\varepsilon^{-1} t & \text { if } t \in[0, \varepsilon] \\
1 & \text { if } t \in[\varepsilon, \infty)
\end{array}\right.
$$

and let $f_{n}:=e_{n}-e_{n}^{\frac{1}{2}} g_{\varepsilon}\left(e_{n}^{\prime}\right) e_{n}^{\frac{1}{2}}$. The same proof as [37, Proposition 3.3] shows that

$$
\left\|f_{n} \alpha^{j}\left(f_{n}\right)\right\|^{2}<\varepsilon
$$


for any $j=1,2, \ldots, k-1$. For $h \in \operatorname{Ped}(\mathcal{W} \otimes \mathbb{K})_{+, 1}$, we have

$$
\begin{aligned}
\lim _{n \rightarrow \omega}\left\|\pi_{\tau \otimes \operatorname{Tr}}\left(e_{n}-f_{n}\right) \hat{h}\right\|_{2} & =\lim _{n \rightarrow \omega}\left\|\pi_{\tau \otimes \operatorname{Tr}}\left(e_{n}^{\frac{1}{2}} g_{\varepsilon}\left(e_{n}^{\prime}\right) e_{n}^{\frac{1}{2}}\right) \hat{h}\right\|_{2} \\
& \leq \lim _{n \rightarrow \omega}\left\|\pi_{\tau \otimes \operatorname{Tr}}\left(g_{\varepsilon}\left(e_{n}^{\prime}\right) e_{n}^{\frac{1}{2}}\right) \hat{h}\right\|_{2} \\
& =\lim _{n \rightarrow \omega}\left\|\pi_{\tau \otimes \operatorname{Tr}}\left(g_{\varepsilon}\left(e_{n}^{\prime}\right) h^{\frac{1}{2}} e_{n}^{\frac{1}{2}}\right) \widehat{h^{\frac{1}{2}}}\right\|_{2} \\
& \leq \lim _{n \rightarrow \omega}\left\|\pi_{\tau \otimes \operatorname{Tr}}\left(g_{\varepsilon}\left(e_{n}^{\prime}\right)\right) \widehat{h^{\frac{1}{2}}}\right\|_{2}=0 .
\end{aligned}
$$

Hence $\tau_{\omega}\left(\left[\left(f_{n}\right)_{n}\right]\right)=\tau_{\omega}\left(\left[\left(e_{n}\right)_{n}\right]\right)=1 / k$ (see Remark 2.4 $)$. Therefore we obtain the conclusion by the usual diagonal argument.

Lemma 6.3. Let $\alpha$ be a trace scaling automorphism of $\mathcal{W} \otimes \mathbb{K}$. Then for any $k \in \mathbb{N}$, there exists a projection $p$ in $F(\mathcal{W} \otimes \mathbb{K})$ such that

$$
\tau_{\omega}(p)=\frac{1}{k}, \quad p \alpha^{j}(p)=0
$$

for any $j=1,2, \ldots, k-1$, and $p$ is Murray-von Neumann equivalent to $\alpha(p)$

Proof. By Lemma 6.2, there exists a positive contraction $f$ in $F(\mathcal{W} \otimes \mathbb{K})$ such that

$$
\tau_{\omega}(f)=\frac{1}{k}, \quad f \alpha^{j}(f)=0
$$

for any $j=1,2, \ldots, k-1$. Since $f$ is a contraction, $d_{\tau_{\omega}}(f) \geq \tau_{\omega}(f)=1 / k$. Let $\varepsilon>0$. (Note that we may assume $0<1 / k-\varepsilon<1$.) By Proposition 4.2, there exists a projection $p$ in $\overline{f F(\mathcal{W} \otimes \mathbb{K}) f}$ such that $\tau_{\omega}(p)=1 / k-\varepsilon$. It is easy to see that $p \alpha^{j}(p)=0$ for any $j=1,2, \ldots . k-1$. Since the tracial state on $F(\mathcal{W} \otimes \mathbb{K}) \cong$ $F(\mathcal{W})$ is unique by Proposition 4.1, $\tau_{\omega} \circ \alpha=\tau_{\omega}$, and hence $\tau_{\omega}(p)=\tau_{\omega}(\alpha(p))$. Therefore Theorem 5.8 implies that $p$ is Murray-von Neumann equivalent to $\alpha(p)$. Consequently, we obtain the conclusion by the usual diagonal argument.

The following theorem is the main theorem in this section. The proof is based on 25 and $[26$.

Theorem 6.4. Let $\alpha$ be a trace scaling automorphism of $\mathcal{W} \otimes \mathbb{K}$. Then $\alpha$ has the Rohlin property.

Proof. For any $N \geq 2$, it follows from Lemma 6.3 that there exists a (non-unital) homomorphism $\varphi$ from $M_{N}(\mathbb{C})$ to $F(\mathcal{W} \otimes \mathbb{K})$ such that

$$
\alpha\left(\varphi\left(e_{i j}\right)\right)=\varphi\left(e_{i+1 j+1}\right)
$$

for any $i, j=1, \ldots, N-1$, where $\left\{e_{i j}\right\}_{i, j=1}^{N}$ is the standard matrix units of $M_{N}(\mathbb{C})$ (see [25. Lemma 4.3] for details). By the same argument as in the proof of [25, Lemma 2.1] and the usual diagonal argument, we see that for any $m \in \mathbb{N}$, there exists a projection $p$ in $F(\mathcal{W} \otimes \mathbb{K})$ such that

$$
\tau_{\omega}(p)=\frac{1}{m}, \quad p \alpha^{j}(p)=0, \quad \alpha^{m}(p)=p
$$

for any $j=1,2, \ldots, m-1$. Note that there exists an element $v$ in $F(\mathcal{W} \otimes \mathbb{K}) \cong F(\mathcal{W})$ such that

$$
v^{*} v=1-\sum_{j=0}^{m-1} \alpha^{j}(p), \quad v v^{*} \leq p
$$

because $\mathcal{W}$ has property $(\mathrm{SI})$ and we have $\tau_{\omega}\left(1-\sum_{j=0}^{m-1} \alpha^{j}(p)\right)=0$. Therefore the rest of the proof is the same as [25, Theorem 2.1] and [26, Lemma 4.4].

An automorphism $\alpha$ of $\mathcal{W}$ is said to be strongly outer if $\tilde{\alpha}$ is not inner in $\pi_{\tau}(\mathcal{W})^{\prime \prime}$. The same proof as Lemma 6.2 shows the following lemma. 
Lemma 6.5. Let $\alpha$ be an automorphism of $\mathcal{W}$ such that $\alpha^{m}$ is strongly outer for any $m \in \mathbb{Z} \backslash\{0\}$. Then for any $k \in \mathbb{N}$, there exists a positive contraction $f$ in $F(\mathcal{W})$ such that

$$
\tau_{\omega}(f)=\frac{1}{k}, \quad f \alpha^{j}(f)=0
$$

for any $j=1,2, \ldots, k-1$.

Lemma 6.6. Let $\alpha$ be an automorphism of $\mathcal{W}$ such that $\alpha^{m}$ is strongly outer for any $m \in \mathbb{Z} \backslash\{0\}$. Then for any $k \in \mathbb{N}$, there exists a projection $p$ in $F(\mathcal{W})$ such that such that

$$
\tau_{\omega}(p)=\frac{1}{k}, \quad p \alpha^{j}(p)=0
$$

for any $j=1,2, \ldots, k-1$, and $p$ is Murray-von Neumann equivalent to $\alpha(p)$.

Proof. In a similar way as in Lemma 6.3, we see that there exists a projection $p$ in $F(\mathcal{W})$ such that

$$
\tau_{\omega}(p)=\frac{1}{k}, \quad p \alpha^{j}(p)=0
$$

for any $j=1,2, \ldots, k-1$. Since every automorphism of $\mathcal{W}$ is approximately inner by Theorem 2.7, we obtain the conclusion by [35, Lemma 4.3].

By the same arguments as in the proof of Theorem 6.4, we obtain the following theorem.

Theorem 6.7. Let $\alpha$ be an automorphism of $\mathcal{W}$ such that $\alpha^{m}$ is strongly outer for any $m \in \mathbb{Z} \backslash\{0\}$. Then $\alpha$ has the Rohlin property.

\section{OUter CONJUGACY}

In this section we shall classify trace scaling automorphisms of $\mathcal{W} \otimes \mathbb{K}$ up to outer conjugacy. Using Theorem 5.7 instead of [17, Lemma 1], we can prove the following theorem by essentially the same argument as in the proof of [17, Theorem 1]. (See also [18] and [29].)

Theorem 7.1. Let $A$ be a $\mathrm{C}^{*}$-algebra which is isomorphic to $\mathcal{W}$ or $\mathcal{W} \otimes \mathbb{K}$, and let $\alpha$ be an automorphism of $A$ with the Rohlin property. For any unitary element $u$ in $F(A)$, there exists a unitary element $v$ in $F(A)$ such that $u=v \alpha(v)^{*}$.

The following lemma is an immediate consequence of the theorem above and Corollary 4.10

Lemma 7.2. Let $A$ be a $\mathrm{C}^{*}$-algebra which is isomorphic to $\mathcal{W}$ or $\mathcal{W} \otimes \mathbb{K}$, and let $\alpha$ be an automorphism of $A$ with the Rohlin property. Then for any finite subsets $E \subset A^{\sim}, F \subset A$ and $\varepsilon>0$, there exist a finite subset $G \subset A$ and $\delta>0$ such that the following holds. If $u$ is a unitary element in $M(A)$ satisfying

$$
\|[u, a]\|<\delta
$$

for any $a \in G$, then there exists a unitary element $v$ in $A^{\sim}$ such that

$$
\|[v, x]\|<\varepsilon, \quad\left\|\left(u-v \alpha(v)^{*}\right) y\right\|<\varepsilon, \quad\left\|y\left(u-v \alpha(v)^{*}\right)\right\|<\varepsilon
$$

for any $x \in E$ and $y \in F$.

The following theorem is the main theorem in this paper.

Theorem 7.3. Let $\alpha$ and $\beta$ be trace scaling automorphisms of $\mathcal{W} \otimes \mathbb{K}$. Then $\alpha$ and $\beta$ are outer conjugate if and only if $\lambda(\alpha)=\lambda(\beta)$. 
Proof. The only if part is obvious. We will show the if part. Theorem 6.4 implies that $\alpha$ and $\beta$ have the Rohlin property. Since $\lambda(\alpha)=\lambda(\beta), \alpha$ is approximately unitarily equivalent to $\beta$ by Theorem 2.7. Therefore we obtain the conclusion by Lemma 7.2 and the Bratteli-Elliott-Evans-Kishimoto intertwining argument 13 (see also [19], 27], 34, [41] and [51] for similar arguments). Indeed, let $\left\{x_{n}\right\}_{n \in \mathbb{N}}$ be a dense set in the unit ball of $\mathcal{W} \otimes \mathbb{K}$. By induction, we shall construct sequences of automorphisms $\left\{\alpha_{2 n}\right\}_{n=0}^{\infty},\left\{\beta_{2 n+1}\right\}_{n=0}^{\infty}$ of $\mathcal{W} \otimes \mathbb{K}$ and sequences of unitaries $\left\{u_{n}\right\}_{n=0}^{\infty}$, $\left\{v_{n}\right\}_{n=0}^{\infty},\left\{w_{n}\right\}_{n=0}^{\infty},\left\{\widetilde{w}_{n}\right\}_{n=0}^{\infty}$ in $(\mathcal{W} \otimes \mathbb{K})^{\sim}$ as follows: Put $\alpha_{0}:=\alpha, \beta_{1}:=\beta$, and let $F_{1}:=\left\{x_{1}, x_{1}^{*}\right\}, F_{1}^{\prime}:=\left\{x_{1}, x_{1}^{*}\right\}, E_{1}:=\{1\}$. Applying Lemma 7.2 to $\beta_{1}, E_{1}, F_{1}^{\prime}$ and $1 / 2$, we obtain a finite subset $G_{1} \subset \mathcal{W} \otimes \mathbb{K}$ and $\delta_{1}>0$. Set

$$
F_{2}:=\beta_{1}^{-1}\left(G_{1}\right) \cup F_{1} \cup\left\{x_{2}, x_{2}^{*}\right\} .
$$

Since $\beta_{1}$ is approximately unitarily equivalent to $\alpha_{0}$, there exists a unitary element $u_{0}$ in $(\mathcal{W} \otimes \mathbb{K})^{\sim}$ such that

$$
\left\|\beta_{1}(a)-u_{0} \alpha_{0}(a) u_{0}^{*}\right\|<\frac{\delta_{1}}{2}
$$

for any $a \in F_{2}$. Put $\alpha_{2}:=\operatorname{Ad}\left(u_{0}\right) \circ \alpha_{0}, v_{0}:=u_{0}$, and let $w_{0}:=u_{0} \alpha_{0}\left(v_{0}\right) v_{0}^{*}$, $\widetilde{w}_{0}:=w_{0}$. Set

$$
E_{2}:=F_{2} \cup \operatorname{Ad}\left(v_{0}\right)\left(F_{2}\right) \cup\left\{\widetilde{w}_{0}\right\}, \quad F_{2}^{\prime}:=F_{2} \cup F_{2} \widetilde{w}_{0}^{*} .
$$

Applying Lemma 7.2 to $\alpha_{2}, E_{2}, F_{2}^{\prime}$ and $1 / 2^{2}$, we obtain a finite subset $G_{2} \subset \mathcal{W} \otimes \mathbb{K}$ and $\delta_{2}>0$. We may assume that $\delta_{2}<\delta_{1} / 2$. Set

$$
F_{3}:=\alpha_{2}^{-1}\left(G_{2}\right) \cup F_{2} \cup\left\{x_{3}, x_{3}^{*}\right\} .
$$

Since $\alpha_{2}$ is approximately unitarily equivalent to $\beta_{1}$, there exists a unitary element $u_{1}$ in $(\mathcal{W} \otimes \mathbb{K})^{\sim}$ such that

$$
\left\|\alpha_{2}(a)-u_{1} \beta_{1}(a) u_{1}^{*}\right\|<\frac{\delta_{2}}{2}
$$

for any $a \in F_{2}$. Put $\beta_{3}:=\operatorname{Ad}\left(u_{1}\right) \circ \beta_{1}$. By (1) and (2), we have

$$
\left\|\left[u_{1}, a\right]\right\|<\delta_{1}
$$

for any $a \in G_{1}$. Hence there exists a unitary element $v_{1}$ such that

$$
\left\|\left[v_{1}, x\right]\right\|<\frac{1}{2}, \quad\left\|y\left(u_{1}-v_{1} \beta_{1}\left(v_{1}\right)^{*}\right)\right\|<\frac{1}{2}
$$

for any $x \in E_{1}$ and $y \in F_{1}^{\prime}$ by Lemma 7.2 Put $w_{1}:=u_{1} \beta_{1}\left(v_{1}\right) v_{1}^{*}, \widetilde{w}_{1}:=w_{1}$, and set

$$
E_{3}:=F_{3} \cup \operatorname{Ad}\left(v_{1}\right)\left(F_{3}\right) \cup\left\{\widetilde{w}_{1}\right\}, \quad F_{3}^{\prime}:=F_{3} \cup F_{3} \widetilde{w}_{1}^{*} .
$$

Applying Lemma 7.2 to $\beta_{3}, E_{3}, F_{3}^{\prime}$ and $1 / 2^{3}$, we obtain a finite subset $G_{3} \subset \mathcal{W} \otimes \mathbb{K}$ and $\delta_{3}>0$. We may assume that $\delta_{3}<\delta_{2} / 2$. Set

$$
F_{4}:=\beta_{3}^{-1}\left(G_{3}\right) \cup F_{3} \cup\left\{x_{4}, x_{4}^{*}\right\} .
$$

Since $\beta_{3}$ is approximately unitarily equivalent to $\alpha_{2}$, there exists a unitary element $u_{2}$ in $(\mathcal{W} \otimes \mathbb{K})^{\sim}$ such that

$$
\left\|\beta_{3}(a)-u_{2} \alpha_{2}(a) u_{2}^{*}\right\|<\frac{\delta_{3}}{2}
$$

for any $a \in F_{3}$. Put $\alpha_{4}:=\operatorname{Ad}\left(u_{2}\right) \circ \alpha_{2}$. By (2) and (3), we have

$$
\left\|\left[u_{2}, a\right]\right\|<\delta_{2}
$$

for any $a \in G_{2}$. Hence there exists a unitary element $v_{2}$ such that

$$
\left\|\left[v_{2}, x\right]\right\|<\frac{1}{2^{2}}, \quad\left\|y\left(u_{2}-v_{2} \alpha_{2}\left(v_{2}\right)^{*}\right)\right\|<\frac{1}{2^{2}}
$$


for any $x \in E_{2}$ and $y \in F_{2}^{\prime}$ by Lemma 7.2 Put $w_{2}:=u_{2} \alpha_{2}\left(v_{2}\right) v_{2}^{*}$, and let $\widetilde{w}_{2}:=$ $w_{2} v_{2} \widetilde{w}_{0} v_{2}^{*}$. Set

$$
E_{4}:=F_{4} \cup \operatorname{Ad}\left(v_{2} v_{0}\right)\left(F_{4}\right) \cup\left\{\widetilde{w}_{2}\right\}, \quad F_{4}^{\prime}:=F_{4} \cup F_{4} \widetilde{w}_{2}^{*} .
$$

Applying Lemma 7.2 to $\alpha_{4}, E_{4}, F_{4}^{\prime}$ and $1 / 2^{4}$, we obtain a finite subset $G_{4} \subset \mathcal{W} \otimes \mathbb{K}$ and $\delta_{4}>0$. We may assume that $\delta_{4}<\delta_{3} / 2$. Set

$$
F_{5}:=\alpha_{4}^{-1}\left(G_{4}\right) \cup F_{4} \cup\left\{x_{5}, x_{5}^{*}\right\} .
$$

Since $\alpha_{4}$ is approximately unitarily equivalent to $\beta_{3}$, there exists a unitary element $u_{3}$ in $(\mathcal{W} \otimes \mathbb{K})^{\sim}$ such that

$$
\left\|\alpha_{4}(a)-u_{3} \beta_{3}(a) u_{3}^{*}\right\|<\frac{\delta_{4}}{2}
$$

for any $a \in F_{4}$. Put $\beta_{5}:=\operatorname{Ad}\left(u_{3}\right) \circ \beta_{3}$. By (3) and (4), we have

$$
\left\|\left[u_{3}, a\right]\right\|<\delta_{3}
$$

for any $a \in G_{3}$. Hence there exists a unitary element $v_{3}$ such that

$$
\left\|\left[v_{3}, x\right]\right\|<\frac{1}{2^{3}}, \quad\left\|y\left(u_{3}-v_{3} \beta_{3}\left(v_{3}\right)^{*}\right)\right\|<\frac{1}{2^{3}}
$$

for any $x \in E_{3}$ and $y \in F_{3}^{\prime}$ by Lemma 7.2 Put $w_{3}:=u_{3} \beta_{3}\left(v_{3}\right) v_{3}^{*}$, and let $\widetilde{w}_{3}:=$ $w_{3} v_{3} \widetilde{w}_{1} v_{3}^{*}$.

Repeating this process, we obtain sequences $\left\{\alpha_{2 n}\right\}_{n=0}^{\infty},\left\{\beta_{2 n+1}\right\}_{n=0}^{\infty},\left\{u_{n}\right\}_{n=0}^{\infty}$, $\left\{v_{n}\right\}_{n=0}^{\infty},\left\{w_{n}\right\}_{n=0}^{\infty}$ and $\left\{\widetilde{w}_{n}\right\}_{n=0}^{\infty}$ such that

(i) $\alpha_{2 n}=\operatorname{Ad}\left(u_{2 n-2}\right) \circ \alpha_{2 n-2}, \quad \beta_{2 n+1}=\operatorname{Ad}\left(u_{2 n-1}\right) \circ \beta_{2 n-1}$,

(ii) $w_{2 n}=u_{2 n} \alpha_{2 n}\left(v_{2 n}\right) v_{2 n}^{*}, \quad w_{2 n+1}=u_{2 n+1} \beta_{2 n+1}\left(v_{2 n+1}\right) v_{2 n+1}^{*}$,

$\widetilde{w}_{n+1}=w_{n+1} v_{n+1} \widetilde{w}_{n-1} v_{n+1}^{*}$,

(iii) $\left\|\beta_{2 n-1}\left(x_{i}\right)-\alpha_{2 n}\left(x_{i}\right)\right\|<\frac{\delta_{2 n-1}}{2}, \quad 1 \leq \forall i \leq 2 n$,

(iv) $\left\|\alpha_{2 n}\left(x_{i}\right)-\beta_{2 n+1}\left(x_{i}\right)\right\|<\frac{\delta_{2 n}}{2}, \quad 1 \leq \forall i \leq 2 n+1$,

(v) $\left\|\left[v_{2 n}, x_{i}\right]\right\|<\frac{1}{2^{2 n}}, \quad\left\|\left[v_{2 n}, \operatorname{Ad}\left(v_{2 n-2} v_{2 n-4} \cdots v_{0}\right)\left(x_{i}\right)\right]\right\|<\frac{1}{2^{2 n}}$,

$$
\left\|\left[v_{2 n}, \widetilde{w}_{2 n-2}\right]\right\|<\frac{1}{2^{2 n}}, \quad 1 \leq \forall i \leq 2 n,
$$

(vi) $\left\|\left[v_{2 n+1}, x_{i}\right]\right\|<\frac{1}{2^{2 n+1}}, \quad\left\|\left[v_{2 n+1}, \operatorname{Ad}\left(v_{2 n-1} v_{2 n-3} \cdots v_{1}\right)\left(x_{i}\right)\right]\right\|<\frac{1}{2^{2 n+1}}$,

$$
\left\|\left[v_{2 n+1}, \widetilde{w}_{2 n-1}\right]\right\|<\frac{1}{2^{2 n+1}}, \quad 1 \leq \forall i \leq 2 n+1,
$$

(vii) $\left\|x_{i}\left(u_{2 n}-v_{2 n} \alpha_{2 n}\left(v_{2 n}\right)^{*}\right)\right\|<\frac{1}{2^{2 n}}$,

$$
\left\|x_{i}^{*} \widetilde{w}_{2 n-2}^{*}\left(u_{2 n}-v_{2 n} \alpha_{2 n}\left(v_{2 n}\right)^{*}\right)\right\|<\frac{1}{2^{2 n}}, \quad 1 \leq \forall i \leq 2 n,
$$

(viii) $\left\|x_{i}\left(u_{2 n+1}-v_{2 n+1} \beta_{2 n+1}\left(v_{2 n+1}\right)^{*}\right)\right\|<\frac{1}{2^{2 n+1}}$,

$$
\left\|x_{i}^{*} \widetilde{w}_{2 n-1}^{*}\left(u_{2 n+1}-v_{2 n+1} \beta_{2 n+1}\left(v_{2 n+1}\right)^{*}\right)\right\|<\frac{1}{2^{2 n+1}}, \quad 1 \leq \forall i \leq 2 n+1,
$$

for any $n \in \mathbb{N}$, where $\left\{\delta_{n}\right\}_{n \in \mathbb{N}}$ is a sequence of positive numbers such that $\delta_{n}<$ $\delta_{n-1} / 2$.

For any $n \in \mathbb{N}$, define $\theta_{n}:=\operatorname{Ad}\left(v_{2 n} v_{2 n-2} \cdots v_{0}\right)$ and $\gamma_{n}:=\operatorname{Ad}\left(v_{2 n+1} v_{2 n-1} \cdots v_{1}\right)$. By (v), (vi) and the same proof as [19, Theorem 3.5], the point-norm limit maps $\theta=\lim _{n \rightarrow \infty} \theta_{n}$ and $\gamma=\lim _{n \rightarrow \infty} \gamma_{n}$ exist and define automorphisms on $\mathcal{W} \otimes \mathbb{K}$. 
For any $n \in \mathbb{N}$, (ii) and (vii) imply that

$$
\left\|x_{i}\left(w_{2 n}-1\right)\right\|<\frac{1}{2^{2 n}}, \quad\left\|\left(w_{2 n}-1\right) \widetilde{w}_{2 n-2} x_{i}\right\|<\frac{1}{2^{2 n}}
$$

for any $i=1,2 \ldots, 2 n$. By (ii) and (v), we have for any $n \in \mathbb{N}$,

$$
\begin{aligned}
\left\|x_{i}\left(\widetilde{w}_{2 n}-\widetilde{w}_{2 n-2}\right)\right\| & =\left\|x_{i} w_{2 n} v_{2 n} \widetilde{w}_{2 n-2} v_{2 n}^{*}-x_{i} \widetilde{w}_{2 n-2} v_{2 n} v_{2 n}^{*}\right\| \\
& \leq\left\|x_{i} w_{2 n}\left[v_{2 n}, \widetilde{w}_{2 n-2}\right]\right\|+\left\|x_{i}\left(w_{2 n}-1\right) \widetilde{w}_{2 n-2} v_{2 n}\right\| \\
& <\frac{1}{2^{2 n-1}}
\end{aligned}
$$

and

$$
\begin{aligned}
\left\|\left(\widetilde{w}_{2 n}-\widetilde{w}_{2 n-2}\right) x_{i}\right\| & =\left\|w_{2 n} v_{2 n} \widetilde{w}_{2 n-2} v_{2 n}^{*} x_{i}-\widetilde{w}_{2 n-2} v_{2 n} v_{2 n}^{*} x_{i}\right\| \\
& \leq\left\|w_{2 n}\left[v_{2 n}, \widetilde{w}_{2 n-2}\right] v_{2 n}^{*} x_{i}\right\|+\left\|\left(w_{2 n}-1\right) \widetilde{w}_{2 n-2} x_{i}\right\| \\
& <\frac{1}{2^{2 n-1}}
\end{aligned}
$$

for any $i=1,2 \ldots, 2 n$. Therefore $\left\{\widetilde{w}_{2 n}\right\}_{n \in \mathbb{N}}$ is a strict Cauchy sequence of unitaries in $(\mathcal{W} \otimes \mathbb{K})^{\sim}$. Since $M(\mathcal{W} \otimes \mathbb{K})$ is strictly complete, there exists a unitary element $w_{0}^{\prime}$ in $M(\mathcal{W} \otimes \mathbb{K})$ such that $\left\{\widetilde{w}_{2 n}\right\}_{n \in \mathbb{N}}$ converges strictly to $w_{0}^{\prime}$. In a similar way, we see that there exists a unitary element $w_{1}^{\prime}$ in $M(\mathcal{W} \otimes \mathbb{K})$ such that $\left\{\widetilde{w}_{2 n+1}\right\}_{n \in \mathbb{N}}$ converges strictly to $w_{1}^{\prime}$.

It can be easily checked that

$$
\alpha_{2 n+2}=\operatorname{Ad}\left(\widetilde{w}_{2 n}\right) \circ \theta_{n} \circ \alpha \circ \theta_{n}^{-1}, \quad \beta_{2 n+3}=\operatorname{Ad}\left(\widetilde{w}_{2 n+1}\right) \circ \gamma_{n} \circ \beta \circ \gamma_{n}^{-1}
$$

for any $n \in \mathbb{N}$. It follows from (iv) that for any $n \in \mathbb{N}$, we have

$$
\left\|\alpha_{2 n+2}\left(x_{i}\right)-\beta_{2 n+3}\left(x_{i}\right)\right\|<\frac{\delta_{2 n+2}}{2}
$$

for any $i=1,2, \ldots, 2 n+3$. Therefore we see that

$$
\operatorname{Ad}\left(w_{0}^{\prime}\right) \circ \theta \circ \alpha \circ \theta^{-1}(x)=\operatorname{Ad}\left(w_{1}^{\prime}\right) \circ \gamma \circ \beta \circ \gamma^{-1}(x)
$$

for any $x \in \mathcal{W} \otimes \mathbb{K}$ because $\left\{\widetilde{w}_{n}\right\}_{n \in \mathbb{N}}$ is a bounded sequence and $\lim _{n \rightarrow \infty} \delta_{n}=0$.

By Theorem 6.7 and the same proof as above, we obtain the following theorem.

Theorem 7.4. Let $\alpha$ and $\beta$ be automorphisms of $\mathcal{W}$. If $\alpha^{m}$ and $\beta^{m}$ are strongly outer for any $m \in \mathbb{Z} \backslash\{0\}$, then $\alpha$ and $\beta$ are outer conjugate.

\section{ACKNOWLEDGMENTS}

The author would like to thank Hiroki Matui for many helpful discussions and valuable suggestions. He is also grateful to the people in University of Münster, where a part of this work was done, for their hospitality.

\section{REFERENCES}

[1] B. Blackadar, Operator Algebras : Theory of $C^{*}$-Algebras and von Neumann Algebras, Encyclopaedia of Mathematical Sciences, 122, Springer, 2006.

[2] J. Bosa, N. P. Brown, Y. Sato, A. Tikuisis, S. White and W. Winter, Covering dimension of $C^{*}$-algebras and 2-coloured classification, to appear in Mem. Amer. Math. Soc. arXiv:1506.03974 [math.OA]

[3] O. Bratteli and A. Kishimoto, Trace scaling automorphisms of certain stable AF algebras. II, Q. J. Math. 51 (2000), no. 2, 131-154.

[4] A. Connes, Almost periodic states and factors of type $\mathrm{III}_{1}$, J. Functional Analysis 16 (1974), 415-445.

[5] A. Connes, Outer conjugacy classes of automorphisms of factors, Ann. Sci. Ecole Norm. Sup. (4) 8 (1975), no. 3, 383-419.

[6] J. Cuntz, K-theory for certain $C^{*}$-algebras, Ann. of Math. (2) 113 (1981), no. 1, 181-197. 
[7] M. Dadarlat and S. Eilers, Asymptotic unitary equivalence in KK-theory, K -Theory 23 (2001), no. 4, 305-322.

[8] M. Dadarlat and S. Eilers, On the classification of nuclear $C^{*}$-algebras, Proc. London Math. Soc. (3) 85 (2002), no. 1, 168-210.

[9] A. Dean, A continuous field of projectionless $C^{*}$-algebras, Canad. J. Math. 53 (2001), no. 1, $51-72$.

[10] G. A. Elliott, D. E. Evans and A. Kishimoto, Outer conjugacy classes of trace scaling automorphisms of stable UHF algebras, Math. Scand. 83 (1998), no. 1, 74-86.

[11] G. A. Elliott and D. Kucerovsky, An abstract Voiculescu-Brown-Douglas-Fillmore absorption theorem, Pacific J. Math. 198 (2001), no. 2, 385-409.

[12] G. A. Elliott and Z. Niu, The classification of simple separable KK-contractible $C^{*}$-algebras with finite nuclear dimension, preprint, arXiv:1611.05159 [math.OA].

[13] D. E. Evans and A. Kishimoto, Trace scaling automorphisms of certain stable AF algebras, Hokkaido Math. J. 26 (1997), no. 1, 211-224.

[14] J. Gabe, A note on nonunital absorbing extensions, Pacific J. Math. 284 (2016), no. 2, 383-393.

[15] G. Gong and H. Lin, On classification of non-unital simple amenable $C^{*}$-algebras, I, preprint, arXiv:1611.04440 [math.OA]

[16] G. Gong and H. Lin, On classification of non-unital simple amenable $C^{*}$-algebras, II, preprint, arXiv:1702.01073 [math.OA]

[17] R. H. Herman and A. Ocneanu, Stability for integer actions on UHF C*-algebras, J. Funct. Anal. 59 (1984), 132-144.

[18] M. Izumi, The Rohlin property for automorphisms of $C^{*}$-algebras, Mathematical physics in mathematics and physics (Siena, 2000) Fields Inst. Commun., vol. 30, Amer. Math. Soc., Providence, RI, 2001, pp. 191-206.

[19] M. Izumi, Finite group actions on $C^{*}$-algebras with the Rohlin property, I, Duke Math. J. 122 (2004), no. 2, 233-280.

[20] M. Izumi, Group actions on operator algebras, in: Proceedings of the International Congress of Mathematicians, vol. III, Hyderabad, India, 2010, Hindustan Book Agency, 2010, pp. 1528-1548.

[21] B. Jacelon, A simple, monotracial, stably projectionless $C^{*}$-algebra, J. Lond. Math. Soc. (2) 87 (2013), no. 2, 365-383.

[22] E. Kirchberg, Central sequences in $C^{*}$-algebras and strongly purely infinite algebras, Operator Algebras: The Abel Symposium 2004, 175-231, Abel Symp., 1, Springer, Berlin, 2006.

[23] E. Kirchberg and M. Rørdam, Non-simple purely infinite $C^{*}$-algebras, Amer. J. Math. 122 (2000), no.3, 637-666.

[24] E. Kirchberg and M. Rørdam, Central sequence $C^{*}$-algebras and tensorial absorption of the Jiang-Su algebra, J. Reine Angew. Math. 695 (2014), 175-214.

[25] A. Kishimoto, The Rohlin property for automorphisms of UHF algebras, J. Reine Angew. Math. 465 (1995), 183-196.

[26] A.Kishimoto. The Rohlin property for shifts on UHF algebras and automorphisms of Cuntz algebras, J. Funct. Anal. 140 (1996), no. 1, 100-123.

[27] A. Kishimoto, Unbounded derivations in AT algebras, J. Funct. Anal. 160 (1998), no. 1, $270-311$.

[28] A. Kishimoto, Automorphisms of AT algebras with the Rohlin property, J. Operator Theory 40 (1998), no. 2, 277-294.

[29] A. Kishimoto, The Rohlin property for shift automorphisms, Rev. Math. Phys. 12 (2000), no. 7, 965-980

[30] A. Kishimoto and A. Kumjian, Simple stably projectionless $C^{*}$-algebras arising as crossed products, Canad. J. Math. 48 (1996), no. 5, 980-996.

[31] A. Kishimoto and A. Kumjian, Crossed products of Cuntz algebras by quasi-free automorphisms, in Operator algebras and their applications (Waterloo, ON, 1994/1995), 173-192, Fields Inst. Commun., 13, Amer. Math. Soc., Providence, RI, 1997.

[32] H. Lin, Classification of homomorphisms and dynamical systems, Trans. Amer. Math. Soc. 359 (2007), no. 2, 859-895.

[33] T. Masuda and R. Tomatsu, Rohlin flows on von Neumann algebras, Mem. Amer. Math. Soc. 244 (2016), no. 1153.

[34] H. Matui, Classification of outer actions of $\mathbb{Z}^{N}$ on $\mathcal{O}_{2}$, Adv. Math. 217 (2008), no. 6, 28722896.

[35] H. Matui, $\mathbb{Z}$-actions on $A H$ algebras and $\mathbb{Z}^{2}$-actions on AF algebras, Comm. Math. Phys. 297 (2010), no. 2, 529-551.

[36] H. Matui, Classification of homomorphisms into simple $\mathcal{Z}$-stable $C^{*}$-algebras, J. Funct. Anal. 260 (2011), no. 3, 797-831. 
[37] H. Matui and Y. Sato, Z-stability of crossed products by strongly outer actions, Comm. Math. Phys. 314 (2012), no. 1, 193-228.

[38] H. Matui and Y. Sato, Strict comparison and $\mathcal{Z}$-absorption of nuclear $C^{*}$-algebras, Acta Math. 209 (2012), no. 1, 179-196.

[39] H. Matui and Y. Sato, Z-stability of crossed products by strongly outer actions II, Amer. J. Math. 136 (2014), no. 6, 1441-1496.

[40] H. Matui and Y. Sato, Decomposition rank of UHF-absorbing $C^{*}$-algebras, Duke Math. J. 163 (2014), no. 14, 2687-2708.

[41] H. Nakamura, Aperiodic automorphisms of nuclear purely infinite simple $C^{*}$-algebras, Ergodic Theory Dynam. Systems 20 (2000), no. 6, 1749-1765.

[42] N. Nawata, Fundamental group of simple $C^{*}$-algebras with unique trace III, Canad. J. Math. 64, (2012), no. 3, 573-587.

[43] N. Nawata, Picard groups of certain stably projectionless $C^{*}$-algebras, J. Lond. Math. Soc. (2) 88 (2013), no. 2, 161-180.

[44] A. Ocneanu, Actions of discrete amenable groups on von Neumann algebras, Lecture Notes in Mathematics, vol. 1138, Springer-Verlag, Berlin, 1985.

[45] G. K. Pedersen, C ${ }^{*}$-Algebras and Their Automorphism Groups, Academic Press, LondonNew York-San Francisco, 1979

[46] S. Razak, On the classification of simple stably projectionless $C^{*}$-algebras, Canad. J. Math. 54 (2002), no. 1, 138-224.

[47] L. Robert, Classification of inductive limits of 1-dimensional NCCW complexes, Adv. Math. 231 (2012), no. 5, 2802-2836.

[48] L. Robert, Remarks on $\mathcal{Z}$-stable projectionless $C^{*}$-algebras, Glasg. Math. J. 58 (2016), no. 2, 273-277.

[49] M. Rørdam, On the structure of simple $C^{*}$-algebras tensored with a UHF-algebra, J. Funct. Anal. 100 (1991), no. 1, 1-17.

[50] M. Rørdam, The stable and the real rank of $\mathcal{Z}$-absorbing $C^{*}$-algebras, Internat. J. Math. 15 (2004), no. 10, 1065-1084.

[51] Y. Sato, The Rohlin property for automorphisms of the Jiang-Su algebra, J. Funct. Anal. 259 (2010), no. 2, 453-476.

[52] Y. Sato, Discrete amenable group actions on von Neumann algebras and invariant nuclear $C^{*}$-subalgebras, preprint, arXiv:1104.4339v1 [math.OA].

[53] G. Szabó, The classification of Rokhlin flows on $C^{*}$-algebras, preprint, arXiv:1706.09276 [math.OA].

[54] M. Takesaki, Theory of operator algebras. III, Encyclopaedia of Mathematical Sciences, 127. Operator Algebras and Non-commutative Geometry, 8. Springer-Verlag, Berlin, 2003.

Department of Educational Collaboration, Osaka Kyoiku University, 4-698-1 AsahiGAOKA, KashiWARA, OSAKA, 582-8582, JAPAN

E-mail address: nawata@cc.osaka-kyoiku.ac.jp 